\title{
Idempotentes Centrais Primitivos Em Algumas Álgebras de Grupos
}

\author{
Vitor Araujo Garcia \\ DISSERTAÇÃO APRESENTADA \\ $\mathrm{AO}$ \\ Instituto DE MATEMÁticA E EstatísticA \\ DA \\ UNIVERSIDADE DE SÃO PAULO \\ PARA \\ OBTENÇÃO DO TÍTULO \\ $\mathrm{DE}$ \\ Mestre EM CiÊnCIAS \\ Programa: Matemática \\ Orientador: Prof. Dr. Raul Antonio Ferraz
}

Durante o desenvolvimento deste trabalho o autor recebeu auxílio financeiro do CNPq

São Paulo, julho de 2015 


\section{Idempotentes Centrais Primitivos Em Algumas Álgebras de Grupos}

Esta versão da dissertação contém as correções e alterações sugeridas pela Comissão Julgadora durante a defesa da versão original do trabalho, realizada em 25/09/2015. Uma cópia da versão original está disponível no Instituto de Matemática e Estatística da Universidade de São Paulo.

Comissão Julgadora:

- Prof. Dr. Raul Antonio Ferraz (orientador) - IME-USP

- Prof. Dr. Francisco Cesar Polcino Milies - IME-USP

- Prof. Dr. Antonio Paques - UFRGS 


\section{Agradecimentos}

Agradeço a Deus em primeiro lugar.

Também agradeço à minha família e amigos pelo apoio e companhia, sem os qual seria impossível chegar até aqui.

Não posso deixar de agradecer aos professores e colegas, pelo apoio e pelas orientações, em especial agradeço ao professor Raul Antonio Ferraz pela sua paciência e orientação, que sem dúvida não foram apenas de grande ajuda, mas foram essenciais para que este trabalho pudesse ser realizado. 


\section{Resumo}

\section{GARCIA, V. A. Idempotentes Centrais Primitivos em Algumas Álgebras de Gru-}

pos. 2015. Dissertação (Mestrado) - Instituto de Matemática e Estatística, Universidade de São Paulo, São Paulo, 2015.

O objetivo do trabalho é apresentar alguns resultados acerca de anéis de grupos e aplicações, segundo o que foi estudado em livros e artigos sobre o assunto. Inicialmente, apresentaremos alguns fatos básicos sobre anéis de grupos, que podem ser encontrados em [5], e em seguida, apresentaremos os resultados principais, mais recentes, que foram estudados em dois artigos diferentes. No primeiro artigo [4], apresentou-se uma forma de calcular o número de componentes simples de certas álgebras de grupos abelianos finitos, bem como também foi apresentada uma forma de calcular geradores idempotentes de códigos abelianos minimais, suas dimensões e seus pesos. No segundo artigo [2], encontra-se uma descrição feita dos idempotentes centrais primitivos da álgebra de grupo racional de grupos nilpotentes finitos.

Palavras-chave: Anel de Grupo; Idempotentes; Álgebra de Grupo; Código Minimal; Código Abeliano. 


\section{Abstract}

GARCIA, V. A. Primitive Central Idempotents in Some Group Algebras. 2015. Master's Thesis - Instituto de Matemática e Estatística, Universidade de São Paulo, São Paulo, 2015.

Our goal in this project is to present some results about group rings and its applications, as presented in books and articles about this subject. First of all we are going to establish some basic fact about group rings, which can be found mainly in [5], and then we will present the main results, which are more recent, and have been studied in two different articles. In [4], the authors presented a way of evaluating the number of simple components of some finite group algebras, as well presented a way of evaluating idempotent generators of some minimal abelian codes, their dimension and their weights. In [2] there is a complete description of all the primitive central idempotents of the rational group algebra of finite nilpotent groups.

Keywords: Group Rings; Idempotents; Group Algebras; Minimal Code; Abelian Code. 


\section{Sumário}

Lista de Símbolos $\quad$ ix

1 Introdução 1

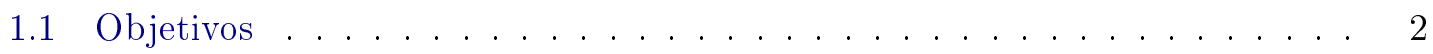

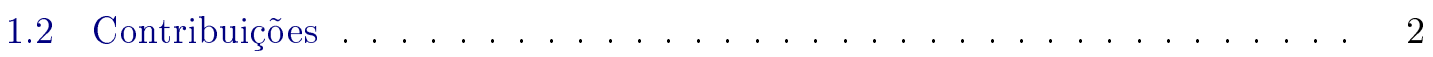

1.3 Organização do Trabalho . . . . . . . . . . . . . . . . . 2

1.4 Considerações Preliminares . . . . . . . . . . . . . . . . 3

1.4 .1 Anéis de Grupos . . . . . . . . . . . . . . . . . 3

1.4.2 Caracteres em Grupos Abelianos Finitos . . . . . . . . . . . . . . . 9

1.4 .3 Códigos Cíclicos . . . . . . . . . . . . . . . . . . 11

1.4 .4 Grupos Nilpotentes . . . . . . . . . . . . . . . . 14

2 Idempotentes e códigos abelianos minimais $\quad 19$

2.1 Considerações Iniciais ． . . . . . . . . . . . . . . . . . . . . . . . . 19

2.2 Quantidade de Componentes Simples . . . . . . . . . . . . . . . . . . 20

2.3 Códigos Cíclicos Minimais . . . . . . . . . . . . . . . . . . . . 24

2.4 Códigos Abelianos Minimais . . . . . . . . . . . . . . . . . . . . . . . . . . . . . . . . . . . 37

2.5 Dimensão e Distância Mínima . . . . . . . . . . . . . . . . . . . 33

2.6 Polinômio Gerador de Códigos Cíclicos Minimais de Tamanho $p^{n} \ldots$. . . . 36

3 Idempotentes Centrais Primitivos em Álgebras de grupo Racionais de Grupos Nilpotentes Finitos $\quad 39$

3.1 Pré-requisitos . . . . . . . . . . . . . . . . . . . . . . 39

3.2 Quando $G$ é Grupo Abeliano Finito . . . . . . . . . . . . . . . . . . . . . . . . . . . . . . . . . . . .

3.3 Grupos Nilpotentes Finitos . . . . . . . . . . . . . . . . . 50

3.4 Calculando um Exemplo concreto . . . . . . . . . . . . . . . . . . 59

4 Conclusões $\quad 65$

4.1 Considerações Finais . . . . . . . . . . . . . . . . . . . . . . 65

$\begin{array}{lll}5 & \text { Referências } & 67\end{array}$

$\begin{array}{ll}\text { Índice Remissivo } & 69\end{array}$ 
viii SUMÁRIO 


\title{
Lista de Símbolos
}

\author{
$H \leq K \quad H$ subgrupo de $K$ \\ $\langle g\rangle \quad$ Grupo gerado por $g$ \\ $U\left(\mathbb{Z}_{n}\right) \quad$ Grupo multiplicativo das unidades de $Z_{n}$ \\ $o(g) \quad$ Ordem do elemento $g$ \\ $\mathbb{C} \quad$ Corpo dos números complexos \\ $\mathbb{Q} \quad$ Corpo dos números racionais \\ $F_{q} \quad$ Corpo finito com $q$ eleentos \\ $C_{G}(H) \quad$ Centralizador de $H$ em $G$ \\ $C_{g} \quad$ Conjunto dos $G$ conjugados de $g$ \\ $Z(G) \quad$ Centro de $G$ \\ $Z_{i}(G) \quad$ i-ésimo centro de $G$ \\ $[h, k] \quad$ Comutador de $h,, k$. O mesmo que escrever $h k h^{-1} k^{-1}$ \\ $h^{g} \quad$ o mesmo que escrever $g^{-1} h g$ \\ $U T(n, F) \quad$ Matrizes $n \times n$ unitriangulares com entradas no corpo $F$ \\ $\mathcal{M}(G) \quad$ Conjunto de todos os subgrupos normais minimais do grupo $G$ \\ $\widehat{H} \quad \frac{1}{|H|} \sum_{g \in H} g$ \\ $\varepsilon(G) \quad \prod_{M \in \mathcal{M}(G)}(1-\widehat{M})$, se $G \neq\{1\}$, e $\varepsilon(\{1\})=1$ \\ $\varepsilon(G, N) \quad \prod_{\bar{M} \in \mathcal{M}(G / N)}(\widehat{N}-\widehat{M})$, se $N$ é subgrupo próprio de $G$, e $\varepsilon(G, G)=\widehat{G}$
}




\section{Capítulo 1}

\section{Introdução}

Nesta dissertação estudaremos em detalhes os artigos Idempotents in group algebras and minimal abelian codes [4] escrito por Ferraz, R. A., e Polcino Milies, C., e Central idempotents in the rational group algebra of a finite nilpotent group [2] escrito por Jespers, E., Leal, G., e Paques, A..

O primeiro artigo trata de idempotentes primitivos e componentes simples de certas álgebras de grupos abelianos finitos sobre corpos finitos e da dimensão de ideais minimais de alguns códigos e será abordado no Capítulo 2.

O segundo artigo traz uma identificação de todos os idempotentes centrais primitivos de álgebras racionais de grupos nilpotentes finitos, começando pelo caso abeliano. Também faremos um exemplo simples para mostrar uma aplicação do resultado principal deste segundo artigo. Será abordado no Capítulo 3.

Neste capítulo introdutório, enunciaremos alguns resultados básicos de que precisaremos como pré-requisitos para as partes principais do trabalho, e deixaremos os demais pré-requisitos para os capítulos seguintes.

Na subseção 1.1.1, enunciaremos alguns resultados e algumas definições básicas da teoria de anéis de grupos de que precisaremos nos capítulos seguintes.

Na subseção 1.1.2, nosso objetivo principal será chegar em um resultado conhecido que relaciona subgrupos e quocientes de grupos abelianos finitos, usando para isso grupos de caracteres. Este resultado será usado no Capítulo 2.

Na subseção 1.1.3, listaremos algumas definições e propriedades básicas sobre códigos cíclicos, bem como sua relação com anéis de polinômios e anéis de grupos. 
Também usaremos essas informações no Capítulo 2.

Na subseção 1.1.4, falaremos sobre grupos nilpotentes e séries centrais, com alguns resultados importantes que serão usados no Capítulo 3. Também apresentaremos uma classe de grupos nilpotentes bem conhecida, como exemplo.

Neste capítulo, aqueles resultados que se encontram em referências não serão provados, mas citaremos onde encontrar as suas demonstrações.

\subsection{Objetivos}

O objetivo deste trabalho é estudar em detalhes os artigos [4] e [2], ambos tratando sobre idempotentes centrais primitivos em algumas álgebras de grupo. Para isso, utilizaremos os conceitos e resultados que foram apresentados no sub-tópico anterior.

Estudar esses artigos permitirá pensar em investigações futuras a respeito do assunto anéis de grupos, bem como permitirá estudar outros artigos mais avançados a respeito do tema.

\subsection{Contribuições}

As principais contribuições deste trabalho foram detalhar (em idioma português) dois artigos matemáticos relativamente recentes, bem como apresentar alguns prérequisitos básicos da teoria e colocá-los numa ordem em que se possa entender os artigos, se estudados como apresentados desde a introdução até o Capítulo 3.

Obs.: Não foi desenvolvido nenhum resultado original/inédito na elaboração desta dissertação.

\subsection{Organização do Trabalho}

No capítulo introdutório, como já dito, o conteúdo principal foi explicar qual o objetivo da dissertação e apresentar uma série de resultados e conceitos básicos que servem como pré-requisitos para os artigos seguintes (principais). As referências que utilizamos para escrever este capítulo foram [5], [1], [9], [8] e [7]. 
No Capítulo 2, apresentaremos e provaremos em detalhes os resultados do artigo [4], que estuda certas álgebras de grupo comutativas sobre corpos finitos. Este artigo será nossa principal referência para este capítulo - também utilizaremos resultados que foram apresentados no capítulo introdutório.

No Capítulo 3, apresentaremos e provaremos os resultados do artigo [2], que apresenta uma caracterização dos idempotentes centrais primitivos da álgebra de grupo racional de grupos nilpotentes finitos.

\subsection{Considerações Preliminares}

Listaremos a seguir algumas noções e resultados básicos que serão usados ao longo dos capítulos principais (capítulos 2 e 3). Os resultados citados a seguir são bem conhecidos, e a maior parte deles não serão provados aqui. No entanto, há referências para textos em que se pode encontrar a prova para eles. Os resultados para os que não há referência serão provados, ou terão um pequeno esboço de prova.

\subsubsection{Anéis de Grupos}

Nesta subseção, introduziremos alguns dos conceitos e resultados básicos da teoria de anéis de grupos, nos baseando principalmente em [5] e [1].

Definição 1.4.1 Seja $G$ um grupo e $R$ um anel com 1 . Definimos $R G$ como sendo o conjunto de todas as somas formais do tipo

$$
\sum_{g \in G} a_{g} g
$$

onde $a_{g} \in R, g \in G$, e apenas uma quantidade finita dos $a_{g}$ 's é diferente de zero. Definimos as operações em $R G$ por:

$$
\sum_{g \in G} a_{g} g+\sum_{g \in G} b_{g} g=\sum_{g \in G}\left(a_{g}+b_{g}\right) g
$$

Se $\alpha=\sum_{g \in G} a_{g} g$ e $\beta=\sum_{g \in G} b_{g} g$ : 


$$
\alpha \beta=\sum_{g, h \in G} a_{g} b_{h} g h .
$$

Com estas operações, RG é chamado anel de grupo de G sobre $R$.

Com as operações acima definidas, temos que $R G$ é um anel com $1=\sum_{g \in G} u_{g} g$, onde $u_{1_{G}}=1$ e, se $g \neq 1_{G}, u_{g}=0$.

Definimos o suporte de $\alpha$ : $\operatorname{supp}(\alpha)=\left\{g \in G: a_{g} \neq 0\right\}$.

Segue da definição que, dados dois elementos em $R G, \alpha=\sum_{g \in G} a_{g} g$ e $\beta=$ $\sum_{g \in G} b_{g} g$, então $\alpha=\beta$ se, e somente se $a_{g}=b_{g}$, para todo $g \in G$.

Se $R$ for comutativo, também podemos definir, para $\lambda \in R$ e $\alpha=\sum_{g \in G} a_{g} g \in$ $R G$, o produto

$$
\lambda \alpha=\sum_{g \in G} \lambda a_{g} g
$$

e com esta operação, temos naturalmente que $R G$ é um $R$-módulo. Neste caso, $R G$ também é chamado álgebra de grupo de $G$ sobre $R$.

Existe uma aplicação injetora natural de $G$ em $R G$, dada por $h \mapsto \sum_{g \in G} a_{g} g$, onde $a_{g}=0$, se $g \neq h$, e $a_{h}=1$ e, com esta aplicação, podemos ver $G$ como um subconjunto de $R G$. Desta forma, podemos dizer que $R G$ é módulo livre, onde $G$ é uma base. Temos, em particular, que se $R$ for comutativo, então a dimensão de $R G$ sobre $R$ está bem definida, e será a cardinalidade de $G$ (se $G$ for finito, será $|\mathrm{G}|$ ).

Também existe aplicação injetora natural de $R$ em $R G$, dada por $r \mapsto \sum_{g \in G} a_{g} g$, onde $a_{1_{G}}=r$ e, se $g \neq 1_{G}$ então $a_{g}=0$. Esta aplicação é um homomorfismo e, portanto, podemos, através dela, enxergar $R$ como um sub-anel de $R G$.

Se $R$ é anel comutativo e $G, H$ são grupos, então

$$
R(G \times H) \simeq(R G) H
$$

(podemos supor que $G \times H$ se trata de produto direto interno) e isso segue do fato de que a função $\varphi: R(G \times H) \rightarrow(R G) H$, dada por 


$$
\varphi\left(\sum_{g h \in G \times H} \alpha_{g h} g h\right)=\sum_{h \in H}\left(\sum_{g \in G} \alpha_{g h} g\right) h
$$

é um isomorfismo.

Agora, enunciaremos uma propriedade universal de anéis de grupos:

Proposição 1.4.2 Seja $G$ um grupo e $R$ um anel. Dados qualquer anel $A$ tal que $R \subset A$ e qualquer função $f: G \rightarrow A$ tal que $f(g h)=f(g) f(h)$, para todos $g, h \in G$, então existe um único homomorfismo de anéis $f^{*}: R G \rightarrow A$ que é R-linear, e tal que $f^{*} \circ i=f$, onde $i: G \rightarrow R G$ é a inclusão dada acima, ou seja, tal que o seguinte diagrama comuta:

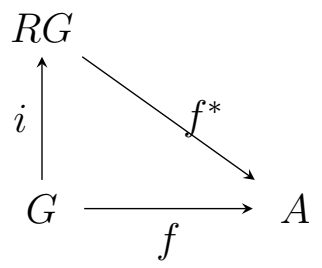

Além disso, se $R$ é central em A (e então A pode ser visto como uma R-álgebra), então $f^{*}$ é um homomorfismo de R-álgebras.

Prova: veja Proposição 3.2 .7 de [5].

O seguinte resultado é um caso particular da proposição acima e, vamos enunciálo separadamente porque precisaremos dele mais adiante.

Corolário 1.4.3 Seja $f: G \rightarrow H$ um homomorfismo de grupos. Então existe um único homomorfismo de anéis $f^{*}: R G \rightarrow R H$ tal que $f^{*}(g)=f(g)$, para todo $g \in G$. Além disso, se $R$ é comutativo, então $f^{*}$ é um homomorfismo de R-álgebras; mais ainda, se $f$ é um epimorfismo (monomorfismo) então $f^{*}$ também é um epimorfismo (monomorfismo).

Prova: veja o Corolário 3.2.8 de [5].

Precisaremos de resultados acerca de anéis de grupos semisimples para estudarmos os capítulos principais. Para chegar nesses resultados, [5] utiliza alguns fatos 
sobre ideais de aumento:

Definição 1.4.4 O homomorfismo $\varepsilon: R G \rightarrow R$ dado por

$$
\varepsilon\left(\sum_{g \in G} a_{g} g\right)=\sum_{g \in G} a_{g}
$$

é chamado de função de aumento de $R G$, e seu núcleo, denotado por $\Delta(G)$, é chamado ideal de aumento de $R G$.

Chegamos então no conhecido teorema que determina condições necessárias e suficientes para um anel de grupo ser semisimples:

Teorema 1.4.5 (Maschke) Seja $G$ um grupo. Então o anel de grupo RG é semisimples se, e somente se valem as seguintes condições:

(i) $R$ é um anel semisimples;

(ii) G é finito;

(iii) $|G|$ é inversivel em $R$.

Prova: ver subseção 3.4 de [5] e, para pré-requisitos sobre ideais de aumento, veja a subseção 3.3 de [5].

Temos, em particular, que se $R=K$ é um corpo e $G$ é um grupo finito, então $R G=K G$ é semisimples se, e somente se $\operatorname{car}(K) \bigwedge|G|$. Portanto, sempre que $K$ for um corpo de característica zero e $G$ for um grupo finito, teremos que $K G$ é semissimples.

É bem conhecido o teorema de Wedderburn-Artin, para anéis semissimples. Enunciemos este teorema para o contexto de anéis de grupos: 
Teorema 1.4.6 Seja $G$ um grupo finito e $K$ um corpo tal que char $(K) \backslash|G|$. Então:

(i) $K G$ é a soma direta de uma quantidade finita de ideais bilaterais $\left\{B_{i}\right\}_{1 \leq i \leq r}$, os componentes simples de KG. Cada $B_{i}$ é um anel simples.

(ii) Qualquer ideal bilateral de KG é a soma direta de alguns membros da família $\left\{B_{i}\right\}_{1 \leq i \leq r}$.

(iii) Cada $B_{i}$ é isomorfo a um anel de matrizes da forma $M_{n_{i}}\left(D_{i}\right)$, onde $D_{i}$ é um anel com divisão que contém uma cópia de $K$ no seu centro.

E o isomorfismo

$$
K G \stackrel{\varphi}{\simeq} \oplus_{i=1}^{r} M_{n_{i}}\left(D_{i}\right)
$$

é um isomorfismo de K-álgebras.

(iv) Em cada anel de matrizes $M_{n_{i}}\left(D_{i}\right)$, o conjunto $I_{i, j}$ das matrizes cujas entradas fora da coluna $j$ são todas nulas é ideal minimal à esquerda e, além disso, $M_{n_{i}}\left(D_{i}\right)=\oplus_{j=1}^{n_{i}} I_{i, j}$.

Além disso, dado $x \in K G$, considerando $\varphi(x)=\left(\alpha_{1}, \ldots, \alpha_{r}\right) \in \oplus_{i=1}^{r} M_{n_{i}}\left(D_{i}\right)$ e definindo $x m_{i}=\alpha_{i} m_{i}$, para todo $m_{i} \in I_{i, j}$, então $I_{i, j}$ pode ser visto como um $K G$-módulo simples.

(v) $I_{i, j} \simeq I_{k, l}$ se, e somente se $i=k$.

(vi) Qualquer KG-módulo simples é isomorfo a algum $I_{i, j}$.

Também temos uma versão do Teorema 2.6.9 de [5], que será muito importante nos capítulos seguintes:

Teorema 1.4.7 Seja $K G=\oplus_{i=1}^{r} B_{i}$ uma decomposição de $K G$ (semisimples) como soma direta de ideais bilaterais minimais da álgebra de grupo KG. Então existe uma familia $\left\{e_{1}, \ldots, e_{r}\right\}$ de elementos de $K G$ tais que: 
(i) $e_{i} \neq 0$ é idempotente e central, $1 \leq i \leq r$;

(ii) Se $i \neq j$, então $e_{i} e_{j}=0$;

(iii) $1=e_{1}+\ldots+e_{r}$;

(iv) $e_{i}$ não pode ser escrito como a soma de dois elementos não-nulos idempotentes centrais de $K G$ que sejam ortogonais.

Este último motiva a seguinte definição:

Definição 1.4.8 Os elementos $e_{i}$ conforme o teorema acima são chamados idempotentes centrais primitivos de $K G$.

Se $K G$ for semissimples temos, pelo Teorema de Wedderburn-Artin que

$$
K G \simeq M_{n_{1}}\left(D_{1}\right) \oplus \ldots \oplus M_{n_{r}}\left(D_{r}\right)
$$

e, pela demonstração do teorema acima, temos que os idempotentes centrais primitivos de $K G$ são relacionados às matrizes identidade, isto é, os idempotentes centrais primitivos $e_{1}, \ldots, e_{r}$ são levados pelo isomorfismo nas matrizes identidade de $M_{n_{1}}\left(D_{1}\right), \ldots, M_{n_{r}}\left(D_{r}\right)$, respectivamente. Segue que todo idempotente central não nulo é soma de idempotentes primitivos centrais (pois os únicos elementos centrais de cada anel de matrizes acima são as matrizes escalares, e só são idempotentes se forem identidades).

Quando $G$ é um grupo abeliano finito, temos ainda o seguinte teorema:

Teorema 1.4 .9 (Perlis-Walker) Seja $G$ um grupo abeliano finito, de ordem $n, e$ seja K um corpo tal que char $(K) \backslash$ n. Então:

$$
K G \simeq \oplus_{d \mid n} a_{d} K\left(\zeta_{d}\right)
$$

onde $\zeta_{d}$ é uma raiz primitiva da unidade de ordem $d$ e $a_{d}=\frac{n_{d}}{\left[K\left(\zeta_{d}\right): K\right]}$. Nesta fórmula, $n_{d}$ denota a quantidade de elementos de ordem d em $G$. 
Em particular, se $K=\mathbb{Q}$, então $a_{d}$ é o número de subgrupos cíclicos de ordem d em $G$.

Obs.: a parte 'em particular' nós temos do fato de que $\left[\mathbb{Q}\left(\zeta_{d}\right): \mathbb{Q}\right]=\phi(d)$, o número de geradores do grupo cíclico de ordem d.

Prova: ver subseção 3.5 de [ 5 ].

Como corpos são anéis simples, temos que o teorema acima nos dá as componentes simples destes anéis de grupos. A parte 'em particular' do teorema nos diz ainda que, se $G$ é um grupo abeliano finito, então a quantidade de idempotentes (centrais) primitivos de $\mathbb{Q} G$ é igual à quantidade de subgrupos cíclicos de $G$.

Na subseção seguinte, estudaremos um pouco sobre a teoria de caracteres em grupos abelianos finitos e, como resultado principal, relacionaremos a quantidade de subgrupos cíclicos de um grupo abeliano finito com a quantidade de fatores cíclicos.

\subsubsection{Caracteres em Grupos Abelianos Finitos}

O objetivo desta subseção é apresentar um teorema sobre teoria de caracteres em grupos abelianos finitos que culmina mostrando que, dado um grupo abeliano finito $G$, existe uma bijeção entre seus subgrupos cíclicos e seus quocientes cíclicos que preserva ordem, pois usaremos este resultado no capítulo 2. Usaremos, para isso, o Capítulo 10 de [ 9 ].

Uma definição possível para o grupo de caracteres de um grupo abeliano finito é a seguinte:

Definição 1.4.10 Seja $G$ um grupo abeliano finito. Então o seu grupo de caracteres $G^{*} \dot{e}$

$$
G^{*}=\operatorname{Hom}\left(G, \mathbb{C}^{\times}\right)
$$

e para quaisquer $f, h \in G^{*}$, definimos o produto $(f h)(g)=f(g) h(g)$, para todo $g \in G$. Desta forma, temos que $G^{*}$ é um grupo. 
Temos o seguinte resultado:

Teorema 1.4.11 Se $G$ é um grupo abeliano finito, então $G \simeq G^{*}$.

Prova: ver Teorema 10.56 de $[\mathbf{9}]$.

Definição 1.4.12 Seja $G$ um grupo abeliano finito. Para cada $x \in G$ definimos o homomorfismo $E_{x}: G^{*} \rightarrow \mathbb{C}^{\times}$por $E_{x}(\varphi)=\varphi(x)$; assim, $E_{x} \in G^{* *}$, e definimos a função $E: G \rightarrow G^{* *}$ por $x \mapsto E_{x}$.

Teorema 1.4.13 Se G é um grupo abeliano finito, então a função E definida acima é um isomorfismo.

Prova: ver Teorema 10.59 de $[\mathbf{9}]$.

Seja $S$ um subgrupo de $G$. Defina $S^{\perp}=\left\{f \in G^{*}: f(s)=1\right.$, para todo $\left.s \in S\right\}$. Temos que $S^{\perp}$ é subgrupo de $G^{*}$ e, a função $S^{\perp} \rightarrow(G / S)^{*}$ dada por $f \mapsto \widehat{f}$, onde $\widehat{f}(\bar{g})=f(g)$ (está bem definida) é um isomorfismo, isto é, $S^{\perp} \simeq(G / S)^{*} \simeq G / S$. Temos o seguinte lema:

Lema 1.4.14 A função $\psi(S)=S^{\perp}$ é uma bijeção entre subgrupos de $G$ e de $G^{*}$.

Prova: Como $G \simeq G^{*}$, então basta mostrar que esta função é injetora, isto é, que dados $S, H$ subgrupos distintos de $G$, então $S^{\perp} \neq H^{\perp}$. Temos:

$\left(S^{\perp}\right)^{\perp}=\left\{E_{x} \in G^{* *}: E_{x}(f)=f(x)=1\right.$, para todo $\left.f \in S^{\perp}\right\} \simeq\left(G^{*} / S^{\perp}\right)^{*} \simeq G^{*} / S^{\perp}$, e, portanto, $S \subseteq\left(S^{\perp}\right)^{\perp}$ e, $\left|\left(S^{\perp}\right)^{\perp}\right|=|G| /\left|S^{\perp}\right|=|G| /(|G / S|)=|S|$ e, assim, temos que $\left(S^{\perp}\right)^{\perp}=S$ e segue o resultado desejado.

Como corolário, temos o seguinte resultado:

Corolário 1.4.15 Seja $G$ um grupo abeliano finito. Então existe uma bijeção $\phi$ entre os subgrupos de $G$ e os quocientes de $G$, tal que $\phi(S) \simeq S$, para todo $S \leq G$. Em particular, a quantidade de subgrupos de $G$ cíclicos de ordem n é igual à quantidade de quocientes de $G$ cíclicos de ordem n, para todo $n$ inteiro positivo. 
Prova: seja $S \leq G$ um subgrupo. Segue da demonstração do lema anterior e do parágrafo que o precede que $\left(S^{\perp}\right)^{\perp}=S \simeq\left(G^{*} / S^{\perp}\right)$. Pelo Teorema 1.11 temos o resultado.

E assim concluímos esta parte.

\subsubsection{Códigos Cíclicos}

Nesta subseção veremos algumas propriedades sobre códigos cíclicos, bem como sua relação com ideais em anéis de grupos e com certos quocientes de anéis de polinômios.

Definição 1.4.16 Um código linear de comprimento $\boldsymbol{n}$ e dimensão $\boldsymbol{k}$ sobre o corpo $F_{q}$ é um subespaço vetorial $C$ de dimensão $\boldsymbol{k}$ do espaço $F_{q}^{n}$, onde $F_{q}$ é o corpo de ordem q. Um código linear $C$ é dito cíclico se, para todo $\left(c_{0}, \ldots, c_{n-1}\right) \in C$ temos $\left(c_{n-1}, c_{0}, \ldots, c_{n-2}\right) \in C$.

Assim, definimos a aplicação $\sigma: C \rightarrow C$ definida por $\sigma\left(c_{0}, \ldots, c_{n-1}\right)=\left(c_{n-1}, c_{0}, \ldots, c_{n-2}\right)$.

Existe um homomorfismo de anéis injetor $\left(c_{0}, \ldots, c_{n-1}\right) \mapsto c_{0}+c_{1} x+\ldots+c_{n-1} x^{n-1} \in$ $F_{q}[x] /\left(x^{n}-1\right)$, e a imagem deste homomorfismo é um ideal $I$ de $F_{q}[x] /\left(x^{n}-1\right)$. Aplicar a função $\sigma$ em $C$ equivale a multiplicar por $x$ em $F_{q}[x] /\left(x^{n}-1\right)$.

Identificaremos um código cíclico $C$ de comprimento $n$ com o ideal $I$ de $F_{q}[x] /\left(x^{n}-\right.$ 1) correspondente.

Proposição 1.4.17 Seja $C$ um código cíclico de comprimento $n$ e dimensão $k>0$ sobre $F_{q}$. Então existe um único polinômio mônico $g(x) \in F_{q}[x]$ tal que, para todo polinômio $c(x) \in F_{q}[x]$ de grau menor que $n$, temos que $c(x) \in C$ se, e somente se $g(x) \mid c(x)$.

Prova: ver Proposição 8.1 de [8].

Assim, temos que a imagem de $g(x)$ em $F_{q}[x] /\left(x^{n}-1\right)$ está em $C$, e $g(x)$ é o polinômio não-nulo de menor grau que satisfaz isso. Mais ainda, temos que a imagem 
de $g(x)$ em $C$ gera $C$ (olhando como ideal do quociente do anel de polinômios). Isso motiva a seguinte definição:

Definição 1.4.18 O polinômio $g(x)$ conforme descrito na proposição anterior é chamado polinômio gerador do código $C$ (e do ideal I).

Notemos que podemos escrever

$$
C=\{\overline{g(x) u(x)}: \operatorname{grau}(u(x))<n-\operatorname{grau}(g(x))-1\},
$$

portanto, temos que a cardinalidade de $C$ é $q^{(n-\operatorname{grau}(g(x)))}=q^{k}$ e, portanto, $\operatorname{grau}(g(x))=$ $n-k$.

Também temos a seguinte proposição:

Proposição 1.4.19 Se C é um código cíclico de tamanho $n$ e $g(x)$ é seu polinômio gerador, então $g(x) \mid\left(x^{n}-1\right)$.

Prova: ver Proposição 8.2 de [8].

Então, podemos fazer a seguinte definição:

Definição 1.4.20 Com o contexto dado acima, o polinômio $h(x)=\left(x^{n}-1\right) / g(x)$ é chamado polinômio de verificação de $C$ (este polinômio tem grau $k$ ).

A partir de agora, vamos supor que $m d c(n, q)=1$.

Desta forma, temos que o polinômio $x^{n}-1 \in F_{q}[x]$ possui $n$ raízes distintas em um corpo de decomposição e, portanto, temos que $m d c(h(x), g(x))=1$.

Também podemos identificar $C$ com um ideal da álgebra de grupo comutativa $F_{q} C_{n}$, de forma análoga à que fizemos para o quociente no anel de polinômios, da seguinte forma: se $g \in C_{n}$ é gerador, então definimos $f: C \rightarrow F_{q} C_{n}$ por:

$$
f\left(c_{1}, \ldots, c_{n}\right)=\sum_{i=1}^{n} c_{i} g^{i} .
$$

Com a suposição acima, temos que $F_{q} C_{n}$ é semisimples (pelo Teorema de Maschke). Pelos teoremas 1.4.6(ii) e 1.4.7, temos que existe um único idempotente $e \in C$ 
que gera $C$. Seja $e(x) \in F_{q}[x]$ um polinômio tal que $\overline{e(x)} \in F_{q}[x] /\left(x^{n}-1\right)$ corresponde a $e$.

Temos a seguinte proposição, que será usada para calcular o polinômio gerador no capítulo 2:

Proposição 1.4.21 Com as notações acima, temos que $g(x)=m d c\left(x^{n}-1, e(x)\right)$

Prova: seja $h(x)$ o polinômio de verificação de $C$. E sejam $r(x) s(x) \in F_{q}[x]$ tais que

$$
r(x) g(x)+s(x) h(x)=1 .
$$

Multiplicando os dois lados da equação acima por $r(x) g(x)$, temos:

$$
(r(x) g(x))^{2}+r(x) s(x) h(x) g(x)=r(x) g(x)
$$

Como $h(x) g(x)=x^{n}-1$, temos que

$$
\overline{(r(x) g(x))^{2}}=\overline{r(x) g(x)}
$$

ou seja, $r(x) g(x)$ é idempotente.

A primeira equação também dos diz que $m d c(r(x), h(x))=1$. Portanto, como $m d c(g(x), h(x))=1$, temos:

$$
g(x)=m d c(r(x) g(x), h(x) g(x))=m d c\left(r(x) g(x), x^{n}-1\right) .
$$

Além disso, temos que $r(x) g(x)=1-s(x) h(x)$ e, temos que

$(r(x) g(x)) g(x)=g(x)-s(x) h(x) g(x)=g(x)-s(x)\left(x^{n}-1\right) \equiv g(x)\left(\bmod \left(x^{n}-1\right)\right)$

e, portanto, $\overline{r(x) g(x)} \in C$ gera $\overline{g(x)}$, que é gerador de $C$. Segue que $\overline{r(x) g(x)}$ é gerador de $C$.

Como $\overline{r(x) g(x)}$ é idempotente e gerador de $C$, então $r(x) g(x) \equiv e(x)\left(\bmod \left(x^{n}-1\right)\right)$ e temos, portanto:

$$
m d c\left(e(x), x^{n}-1\right)=m d c\left(r(x) g(x), x^{n}-1\right)=g(x),
$$


como queríamos.

\subsubsection{Grupos Nilpotentes}

Definiremos e enunciaremos alguns resultados básicos acerca de grupos nilpotentes e séries centrais que serão usados no Capítulo 3, e daremos um exemplo de um tipo de grupos nilpotentes finitos. Para isso, comecemos definindo alguns conceitos básicos:

Definição 1.4.22 Para $h, k \in G$, definimos seu comutador $[h, k]=h k h^{-1} k^{-1}$.

Para subgrupos $H, K$ de $G$, definimos $[H, K]=\langle[h, k]: h \in H, k \in K\rangle$.

Vamos definir os subgrupos $\gamma_{i}(G)$ de $G$ por indução:

$$
\begin{gathered}
\gamma_{1}(G)=G ; \\
\gamma_{i+1}(G)=\left[\gamma_{i}(G), G\right] .
\end{gathered}
$$

Por indução, temos que cada $\gamma_{i}(G)$ é normal em $G$ e, além disso, $\gamma_{i+1}(G) \leq \gamma_{i}(G)$. Isto possibilita a seguinte definição:

Definição 1.4.23 Chamaremos de série central descendente de G a série

$$
G=\gamma_{1}(G) \geq \gamma_{2}(G) \geq \ldots
$$

Também há outra série de interesse.

Definição 1.4.24 Os centros de ordem superior, que denotaremos por $Z_{i}(G)$, são subgrupos de $G$ definidos indutivamente:

$$
\begin{gathered}
Z_{0}(G)=1 \\
Z_{i+1}(G) / Z_{i}(G)=Z\left(G / Z_{i}(G)\right),
\end{gathered}
$$

isto é, se $\nu_{i}: G \rightarrow G / Z_{i}(G)$ é a projeção natural, então $Z_{i+1}(G)$ é a pré-imagem do centro. 
Também dizemos que $Z_{i}(G)$ é o i-ésimo centro de $G$.

É claro que $Z_{1}(G)=Z(G)$.

E definimos a série central ascendente de $G$ :

$$
1=Z_{0}(G) \leq Z_{1}(G) \leq Z_{2}(G) \leq \cdots
$$

Teorema 1.4.25 Seja G um grupo, então existe um inteiro não-negativo c com $Z_{c}(G)=G$ se, e somente se, $\gamma_{c+1}(G)=1$. Além disso, nesse caso, $\gamma_{i+1}(G) \leq$ $Z_{c-i}(G)$, para todo $i$.

Prova: ver Teorema 5.31 de $[\mathbf{9}]$.

Definição 1.4.26 Um grupo $G$ é dito nilpotente se existe inteiro c tal que $\gamma_{c+1}(G)=$ 1 ou, equivalentemente, $Z_{c}=G$. O menor c que satisfaz isso é chamado classe de nilpotência de $G$.

Temos, por exemplo, que um grupo é nilpotente de classe 1 se, e somente se ele for abeliano.

Os próximos teoremas lidam com subgrupos e quocientes de grupos nilpotentes.

Teorema 1.4.27 Todo subgrupo $H$ de um grupo nilpotente $G$ é nilpotente. Mais ainda, se $G$ é nilpotente de classe c, então $H$ é nilpotente de classe no máximo c.

Prova: ver Teorema 5.35 de $[\mathbf{9}]$.

Teorema 1.4.28 Se $G$ é um grupo nilpotente de classe c e $H \triangleleft G$, então $G / H$ é nilpotente de classe no máximo c.

Prova: ver Teorema 5.36 de $[\mathbf{9}]$.

O próximo teorema será muito útil no capítulo 3:

Teorema 1.4.29 Seja $G$ um grupo nilpotente. Se $\{1\} \neq H \triangleleft G$, então $H \cap Z(G) \neq$ $\{1\}$. 
Prova: ver Teorema 5.41 de $[\mathbf{9}]$.

Teorema 1.4.30 Todo p-grupo finito é nilpotente.

Prova: ver Teorema 5.33 de $[\mathbf{9}]$.

Temos também a seguinte definição:

Definição 1.4.31 Uma série

$$
G=G_{1} \geq G_{2} \geq \ldots \geq G_{n}=1
$$

onde cada $G_{i} \triangleleft G$ e $G_{i} / G_{i+1} \leq Z\left(G / G_{i+1}\right)$ é chamada uma série central.

Por 5.1.9 de [7], temos que, se $G$ for nilpotente, as séries centrais ascendente e descendente são centrais e de mesmo tamanho e, além disso, se todos os subgrupos $G_{i}$ de uma série central de um grupo nilpotente $G$ são distintos, então $\gamma_{i+1}(G) \leq$ $G_{i+1} \leq Z_{n-1-i}$, e portanto $G$ é nilpotente de classe $n-1$.

Obs: para uma justificativa do parágrafo acima, veja 5.1 .9 de [7].

Exemplo: Denotemos por $G=U T\left(n, F_{q}\right)(\operatorname{com} n \geq 2)$ o grupo (multiplicativo) das matrizes $n \times n$ triangulares superiores tais que as entradas da diagonal principal são todas iguais a 1 . Pelo teorema anterior, temos que $U T\left(n, F_{q}\right)$ é grupo nilpotente.

Defina $T_{n}$ como sendo o grupo das matrizes triangulares superiores e $N$ como sendo o ideal de $T_{n}\left(F_{q}\right)$ composto pelas matrizes que têm apenas entradas nulas na diagonal principal e abaixo dela. Temos que $N^{i}$ é o ideal de $T_{n}\left(F_{q}\right)$ das matrizes cujas entradas da diagonal principal e das (i-1) diagonais logo acima desta são todas nulas.

Pela seção 5.1 de [7], temos que

$$
1=U_{n} \leq \ldots \leq U_{1}=G
$$

é uma série central, com $U_{i}=1+N^{i}=\left\{1+x: x \in N^{i}\right\}$.

Temos, pelo que foi observado acima, que $U_{i+1} \leq Z_{n-1-i}$. É bem conhecido o fato de que esta inclusão é, na realidade uma igualdade, ou seja, que $U_{i+1}=Z_{n-1-i}$, 
para todo $i$. Vamos verificar sucintamente este fato, para fins de completude:

Antes, observemos os dois seguintes fatos, que são imediatos:

- Se $A \in U_{i}, B \in U_{j}$, então $A B \in U_{i+j}$;

- Vale que $Z_{1}(G)=U_{n-1}$;

Além disso, seja $A \in G$. $A$ pode ser escrito unicamente como uma soma $A=$ $1+B+C$, onde $B, C \in N$ tais que $B \in N^{i}$ e todas as entradas da matriz $C$ acima da $(i-1)$-ésima diagonal acima da diagonal principal são nulas. Assim, temos que $(1+B) \in U_{i}$ e, portanto, $(1+B)^{-1}=(1+D) \in U_{i}$. Temos:

$$
A(1+B)^{-1}=((1+B)+C)(1+B)^{-1}=1+C(1+D)=1+C+C D,
$$

e $C D \in N^{i+1}$. Portanto, temos por indução que no quociente $G / U_{i}, A=1+C$.

Portanto, no quociente acima, todo elemento tem um representante que tem apenas entradas nulas acima da $(i-1)$-ésima diagonal acima da diagonal principal (chamaremos matrizes desse tipo de matrizes do tipo $i$ ).

Assim, para terminar a prova do que queremos, basta notar que para uma matriz $A \notin U_{i}$ do tipo $i$ sempre há uma matriz $B \notin U_{i}$ do tipo $i$ tal que $A B$ e $B A$ são distintos em alguma entrada abaixo da $i$-ésima diagonal acima da diagonal principal, e usar indução (onde o passo inicial é o segundo fato acima).

Portanto, temos que $U T\left(n, F_{q}\right)$ é um grupo nilpotente de classe $(n-1)$. Em particular, temos que para todo inteiro positivo $m$, sempre há um grupo nilpotente de classe $m$.

Para o exemplo acima ficar mais claro, vamos ver um caso mais concreto: tome$\operatorname{mos} G=U T\left(4, F_{q}\right)$. Então temos: 


$$
\begin{gathered}
G=\left\{\left[\begin{array}{cccc}
1 & a_{12} & a_{13} & a_{14} \\
0 & 1 & a_{23} & a_{24} \\
0 & 0 & 1 & a_{34} \\
0 & 0 & 0 & 1
\end{array}\right]: a_{i j} \in F_{q}\right\}=Z_{3}(G) \\
Z_{2}(G)=\left\{\left[\begin{array}{cccc}
1 & 0 & a_{13} & a_{14} \\
0 & 1 & 0 & a_{24} \\
0 & 0 & 1 & 0 \\
0 & 0 & 0 & 1
\end{array}\right]: a_{i j} \in F_{q}\right\} \\
Z_{1}(G)=\left\{\left[\begin{array}{llll}
1 & 0 & 0 & a_{14} \\
0 & 1 & 0 & 0 \\
0 & 0 & 1 & 0 \\
0 & 0 & 0 & 1
\end{array}\right]: a_{14} \in F_{q}\right\}
\end{gathered}
$$

E temos uma descrição completa da série central ascendente de $G$. 


\section{Capítulo 2}

\section{Idempotentes e códigos abelianos}

\section{minimais}

Neste capítulo, vamos calcular a quantidade de componentes simples de certas álgebras de grupos abelianos finitos sobre corpos finitos. Todas estas álgebras que estudamos são semisimples.

Vamos também calcular idempotentes geradores de códigos abelianos minimais de algumas álgebras, ou seja, seus idempotentes (centrais) primitivos. Também vamos calcular a dimensão e o peso desses códigos.

Todo o capítulo será baseado, principalmente, no trabalho que foi feito por Ferraz, R. e Polcino Milies, C. [4].

\subsection{Considerações Iniciais}

Conforme vimos em 1.1.3, se $F=F_{q}$ é o corpo com $q$ elementos, então os códigos cíclicos de comprimento $m$ sobre $F$ podem ser vistos como ideais em $F[X] /\left(X^{m}-1\right)$ ou em $F C_{m}$, onde $C_{m}$ é o grupo cíclico de ordem $m$.

Definição 2.1.1 Um código abeliano sobre o corpo $F$ é um ideal I na álgebra de grupo FA, onde A é um grupo abeliano finito. Um código abeliano minimal é um ideal I que é minimal.

Como vimos na seção 1.1, se $\operatorname{car} F \backslash|A|$, então $F A$ é semisimples e, portanto, todo ideal minimal é um componente simples $B_{i}=F A e_{i}$ de $F A$, onde $e_{i}$ é um 
idempotente central primitivo de $F A$.

\subsection{Quantidade de Componentes Simples}

Seja $F$ um corpo finito, com $q$ elementos, e seja $A$ um grupo abeliano finito tal que $m d c(q,|A|)=1$. Como vimos, $F A$ é semisimples e, se $\left\{e_{1}, \ldots, e_{r}\right\}$ é o conjunto de idempotentes centrais primitivos de $F A$, temos:

$$
F A=\oplus_{i=1}^{r}(F A) e_{i} \simeq \oplus_{i=1}^{r} F_{i},
$$

onde $F_{i} \simeq(F A) e_{i}, 1 \leq i \leq r$, são extensões finitas de $F$.

Defina:

$$
\mathcal{A}=\oplus_{i=1}^{r} F e_{i}
$$

Note que $F e_{i} \simeq F$ como corpos, sob o isomorfismo $a e_{i} \mapsto a(a \in F)$, e o número $r$, que representa a quantidade de componentes simples de $F A$, também representa a dimensão de $\mathcal{A}$ sobre $F$.

O próximo lema traz uma identificação de quais são os elementos de $F A=$ $\oplus_{i=1}^{r}(F A) e_{i}$ que estão em $\mathcal{A}$ :

Lema 2.2.1 Seja $\alpha \in F A$. Então $\alpha \in \mathcal{A}$ se, e somente se $\alpha^{q}=\alpha$.

Prova: Dado $\alpha \in F A$, podemos escrever $\alpha=\sum_{i=1}^{r} \alpha_{i}$, onde $\alpha_{i}=\alpha e_{i} \in F A e_{i} \simeq$ $F_{i}, 1 \leq i \leq r$. Agora, $\alpha$ é um elemento de $\mathcal{A}$ se, e somente se cada $\alpha_{i}$ está em $F e_{i}$, para cada índice $i$. Como $F e_{i} \simeq F$ e $F A e_{i}$ é uma extensão de $F e_{i}$, isto acontece se, e somente se $\alpha_{i}^{q}=\alpha_{i}$ para todo $i$; isto é, se, e somente se $\alpha^{q}=\alpha$.

Definição 2.2.2 Seja g um elemento do grupo abeliano A. A classe q-ciclotômica de g é o conjunto

$$
S_{g}=\left\{g^{q^{j}}: 0 \leq j \leq t_{g}-1\right\},
$$


onde $t_{g}$ é o menor inteiro positivo tal que

$$
q^{t_{g}} \equiv 1(\bmod o(g))
$$

e o $(g)$ denota a ordem de $g$.

Se $(q, o(g))=1$, sempre haverá um tal $t_{g}$ (pois $\bar{g} \in Z_{o(q)}^{*}$ e, portanto, $\bar{g}^{\phi(o(g))}=\overline{1}$, e se $a \in S_{g}$, então $a^{q^{i}} \in S_{g}$, para todo $i$.

Se $S_{g} \cap S_{h} \neq \emptyset$, então existe $a \in S_{g} \cap S_{h}$. Podemos escrever $a=g^{q^{j}}=h^{q^{i}}$. Temos $a^{q^{t_{g-j}}}=g \in S_{h}$ e, similarmente, $h \in S_{g}$. Logo, $S_{g}=S_{h}$.

Temos, portanto, que se $S_{g} \neq S_{h}$, então $S_{g} \cap S_{h}=\emptyset$. Seja $T=\left\{g_{1}, \ldots, g_{s}\right\}$ um conjunto de representantes das classes $q$-ciclotômicas dos elementos de $A$ (há $s$ q-classes ciclotômicas em $A$ ).

Teorema 2.2.3 Seja F um corpo finito de ordem q, e seja A um grupo abeliano finito tal que $(q,|A|)=1$. Então, a quantidade de componentes simples de $F A$ é igual ao número de classes q-ciclotômicas de A.

Prova: conforme observamos acima, o número de componentes simples de $F A$ é igual à dimensão de $\mathcal{A}$ sobre $F$. Para provarmos o teorema, vamos exibir uma base de $\mathcal{A}$ com $s$ elementos.

Dada uma classe q-ciclotômica $S_{g}$, definimos $\eta_{g}=\sum_{h \in S_{g}} h \in F A$. Vamos provar que $\mathcal{B}=\left\{\eta_{g_{i}}: 1 \leq i \leq s\right\}$ é uma base de $\mathcal{A}$ sobre $F$ :

Como $S_{g} \cap S_{h}=\emptyset$ se $g \neq h$, então temos que $\mathcal{B}$ é conjunto linearmente independente. Para mostrar que o dado conjunto gera $\mathcal{A}$ sobre $F$, observemos que, como $F A$ é um anel de característica $q$, então $\eta_{g_{i}}^{q}=\eta_{g_{i}}, 1 \leq i \leq s$, e portanto, pelo lema anterior, temos que $\mathcal{B} \subset \mathcal{A}$.

Seja $\alpha \in \mathcal{A}=\oplus_{i=1}^{r} F e_{i}$. Usando novamente o lema anterior, temos que $\alpha=\alpha^{q}$. Assim, se $\alpha=\sum_{g \in A} \alpha_{g} g$, nós temos:

$$
\alpha=\sum_{g \in A} \alpha_{g} g=\left(\sum_{g \in A} \alpha_{g} g\right)^{q}=\sum_{g \in A} \alpha_{g}^{q} g^{q} .
$$


Como $\alpha_{g} \in F$, então $\alpha_{g}^{q}=\alpha_{g}$ e temos:

$$
\sum_{g \in A} \alpha_{g} g=\sum_{g \in A} \alpha_{g} g^{q}
$$

Como $g^{q}=h^{q}$ implica $S_{g}=S_{h}$, e a função $g \mapsto g^{q}$ é bijetora em $S_{g}$, temos que esta mesma função é bijetora em $A$.

Assim, para cada $g \in A$, temos que $\alpha_{g}=\alpha_{g^{q}}=\ldots=\alpha_{g^{q^{t}-1}}$ e, portanto, em $\alpha$ cada coeficiente de um elemento de $S_{g}$ é $\alpha_{g}$, e temos:

$$
\alpha=\sum_{g \in T} \alpha_{g} \eta_{g}
$$

e concluímos que $\mathcal{B}$ gera $\mathcal{A}$ como $F$ - espaço vetorial, como queríamos.

Como enunciamos no Teorema 1.4.9, o número de componentes simples da álgebra de grupo racional de um grupo abeliano finito $A$ é igual à quantidade de subgrupos cíclicos de $A$ (e, como vimos em 1.4.2, também é igual ao número de fatores cíclicos).

Note que, se $h \in S_{g}$, então $h=g^{q^{j}}$ para algum $j$. Como $(q, o(g))=1$, então $\left(q^{j}, o(g)\right)=1$, o que implica $\langle g\rangle=\langle h\rangle$. Assim, cada $q$-classe ciclotômica $S_{g}$ é um subconjunto do conjunto $\mathcal{G}_{g}$ de todos os geradores do grupo cíclico gerado por $g$. Então, é claro que o número de subgrupos cíclicos de $A$ é um limitante inferior para o número de componentes simples de $F A$, e este limitante é atingido se, e somente se $S_{g}=\mathcal{G}_{g}$, para todo $g \in A$.

Para inteiros positivos $r$ e $m$, denotemos por $\bar{r} \in \mathbb{Z}_{m}$ a imagem de $r$ no anel dos inteiros módulo $m$. Então,

$$
\mathcal{G}_{g}=\left\{g^{r}:(r, o(g))=1\right\}=\left\{g^{r}: \bar{r} \in U\left(\mathbb{Z}_{o(g)}\right)\right\} .
$$

Antes de enunciar o próximo teorema, vamos ao seguinte lema, que será bem útil:

Lema 2.2.4 Sejam $a, b, q \in \mathbb{Z}, a \mid b, \operatorname{mdc}(q, b)=1$ (e portanto $\operatorname{mdc}(q, a)=1)$, 
e suponha que a imagem de $q$ em $U\left(\mathbb{Z}_{b}\right)$ é um gerador (multiplicativo). Então a imagem de q em $U\left(\mathbb{Z}_{a}\right)$ também é gerador (multiplicativo).

Prova: Seja $x \in \mathbb{Z}$, denotemos por $x_{a}$ a imagem de $x$ em $\mathbb{Z}_{a}$, e por $x_{b}$ a imagem de $x$ em $\mathbb{Z}_{b}$, e suponha que $x_{a} \in U\left(\mathbb{Z}_{a}\right)$.

Seja $p_{1}^{r_{1}} \ldots p_{n}^{r_{n}}=m d c(x, b)$ decomposição em fatores primos do m.d.c entre $x$ e $b$ (onde os $p_{i}{ }^{6}$ s são primos distintos), e sejam $m_{1}, \ldots, m_{n}$ os maiores inteiros tais que $p_{i}^{m_{i}} \mid b$. Vamos provar que $m d c\left(x+\frac{a b}{p_{1}^{m_{1}} \ldots p_{n}^{m_{n}}}, b\right)=1$.

Suponha que $p$ é um número primo que divide este m.d.c.. Temos dois casos:

Primeiro caso: se $p \mid x$, temos que, como $p \mid b$, então $p=p_{i}$, para algum $i$, e $p \mid \frac{a b}{p_{1}^{m_{1}} \ldots p_{n}^{m_{n}}}$. Daí, como $p \nmid \frac{b}{p_{1}^{m_{1}} \ldots p_{n}^{m_{n}}}$, temos que $p \mid a$. Mas isto contradiz o fato de que $a$ e $x$ são primos entre si.

Segundo caso: se $p \nmid x$, então $p \neq p_{i}$, para todo $i$. Como $p \mid b$, temos que $p \mid \frac{b}{\frac{p_{1}^{m} \ldots}{m_{n}} \ldots p_{n}^{m}}$, $\frac{b}{p_{1}^{m_{1}} \ldots p_{n}^{m_{n}}} \mid \frac{a b}{p_{1}^{m_{1}} \ldots p_{n}^{m_{n}}}$, e $p \mid\left(x+\frac{a b}{p_{1}^{m_{1}} \ldots p_{n}^{m_{n}}}\right)$. Portanto temos que $p \mid x$, uma contradição.

Assim, por hipótese, temos que existe $m \in \mathbb{Z}$ tal que $q^{m} \equiv x+\frac{a b}{p_{1}^{m_{1}} \ldots p_{n}^{m_{n}}}(\bmod b)$. Como $a \mid b$, temos $q^{m} \equiv x+\frac{a b}{p_{1}^{m_{1}} \ldots p_{n}^{m_{n}}} \equiv x(\bmod a)$. Como x foi pego arbitrariamente dentre os relativamente primos com $a$, temos que $q$ gera o grupo $U\left(\mathbb{Z}_{a}\right)$.

Usaremos este lema para provar o seguinte teorema:

Teorema 2.2.5 Seja F um corpo finito com q elementos, e seja A um grupo abeliano finito, de expoente e, tal que $(q,|A|)=1$. Então $S_{g}=\mathcal{G}_{g}$, para todo $g \in A$ se, e somente se $U\left(\mathbb{Z}_{e}\right)$ é um grupo cíclico gerado por $\bar{q} \in \mathbb{Z}_{e}$.

Prova: Suponha primeiro que $U\left(\mathbb{Z}_{e}\right)$ é cíclico gerado por $\bar{q}$. Para $g \in A$, temos que $o(g) \mid e$ e, pelo lema anterior, $(\bar{q}) \in \mathbb{Z}_{o(g)}$ é um gerador de $U\left(\mathbb{Z}_{o(g)}\right)$.

Para todo elemento $h \in \mathcal{G}_{g}$ temos que $h=g^{r}$, para algum inteiro positivo $r$ tal que $\bar{r} \in U\left(\mathbb{Z}_{o(g)}\right)$, então $\bar{r}=\bar{q}^{j}$, para algum inteiro positivo $j$ e $h=g^{q^{j}} \in S_{g}$. Isso mostra que $\mathcal{G}_{g} \subset S_{g}$. Como a inclusão oposta sempre vale, conforme observado anteriormente, então temos igualdade. 
Por outro lado, suponha que $\mathcal{G}_{g}=S_{g}$, para todo $g \in A$. Como $A$ é abeliano de expoente $e$, então existe $g_{0} \in A$ tal que $o\left(g_{0}\right)=e$ e, em particular, $\mathcal{G}_{g_{0}}=S_{g_{0}}$ (este último por hipótese). Então, para cada inteiro $r$ tal que $\bar{r} \in U\left(\mathbb{Z}_{e}\right)$, temos que $g_{0}^{r} \in S_{g_{0}}$, e portanto existe um inteiro $j$ tal que $\bar{r}=\bar{q}^{j}$. Isso mostra que $\bar{q}$ gera $U\left(\mathbb{Z}_{e}\right)$, como queríamos.

É bem conhecido o fato de que $U\left(\mathbb{Z}_{e}\right)$ é cíclico se, e somente se, $e=2,4, p^{n}$ ou $2 p^{n}$, onde $p$ é um número primo ímpar, e $n$ é um inteiro positivo (ver, por exemplo, Teorema 2.41 de [3]).

Além disso, se $q$ é ímpar, então $q$ gera $U\left(\mathbb{Z}_{2}\right)$; se $q \equiv 3(\bmod 4)$ então $q$ gera $U\left(\mathbb{Z}_{4}\right)$; e $q$ gera $U\left(\mathbb{Z}_{e}\right)$, com $e=p^{n}$ ou $e=2 p^{n}$ se, e somente se $o(q)=\phi\left(p^{n}\right)$ em $U\left(\mathbb{Z}_{e}\right)$.

Temos, portanto, o seguinte corolário:

Corolário 2.2.6 Seja F um corpo finito com q elementos, e seja A um grupo abeliano finito, de expoente e. Então $\mathcal{G}_{g}=S_{g}$ para todo $g \in A$ se, e somente se vale um dos seguintes:

(i) $e=2$ e q é ímpar;

(ii) $e=4$ e $q \equiv 3(\bmod 4)$

(iii) $e=p^{n}$, onde p é um primo ímpar e o(q)= $\phi\left(p^{n}\right)$ em $U\left(\mathbb{Z}_{e}\right)$;

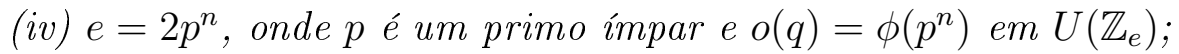

\subsection{Códigos Cíclicos Minimais}

O objetivo desta subseção é calcular os idempotentes primitivos de álgebras de grupos cíclicos do tipo listado no corolário anterior.

Seja $H$ um subgrupo de um grupo $G$. Se $(q,|H|)=1$, definimos

$$
\widehat{H}=\frac{1}{|H|} \sum_{g \in H} g
$$


Temos que $\widehat{H}$ está bem definido e é idempotente em $F G$ (lema 3.6.6 de [5]).

Lema 2.3.1 Seja F um corpo finito com q elementos, e seja $p$ um número primo $e$ $A=\langle a\rangle$ um grupo cíclico de ordem $p^{n}, n \geq 1$. Seja

$$
A=A_{0} \supset A_{1} \supset \ldots \supset A_{n}=1
$$

a cadeia decrescente de todos os subgrupos de $A\left(A_{i}=\left\langle a^{p^{i}}\right\rangle\right)$. Então os elementos

$$
e_{0}=\widehat{A}, e_{i}=\widehat{A_{i}}-\widehat{A_{i-1}}, 1 \leq i \leq n,
$$

forma um conjunto de idempotentes ortogonais de $F A$ tais que $e_{0}+e_{1}+\ldots+e_{n}=1$.

A prova a seguir é encontrada no Lema VII.1.2 de [1] e, logo em seguida, é feita uma observação de que, no caso de $F$ ser finito, estes idempotentes podem não ser primitivos:

Prova: Como $e_{0} \widehat{A_{i}}=\widehat{A_{i}}$, para todo $i$, então nós temos $e_{0} e_{i}=0$, para todo $i>0$. Além disso, se $1 \leq i \leq j, \widehat{A_{i}} \widehat{A_{j}}=A_{i}$, então

$$
\begin{gathered}
e_{i} e_{j}=\left(\widehat{A_{i}}-\widehat{A_{i-1}}\right)\left(\widehat{A_{j}}-\widehat{A_{j-1}}\right)= \\
=\widehat{A_{i}}-\widehat{A_{i}} \widehat{A_{j-1}}-\widehat{A_{i-1}}+\widehat{A_{i-1}}=\widehat{A_{i}}-\widehat{A_{i}} \widehat{A_{j-1}}
\end{gathered}
$$

que é 0 se $i<j$ e $e_{i}$ se $i=j$. Segue que $e_{0}, \ldots, e_{m}$ são $(n+1)$ idempotentes (centrais) dois a dois ortogonais. Além disso, observamos que a soma dos elementos é telescópica, e portanto a soma $\sum_{i=0}^{j} e_{i}=\widehat{A_{j}}$, o que imlplica que a soma de todos esses idempotentes é 1 , como queríamos.

Se $F=\mathbb{Q}$, então temos que esses elementos são os idempotentes primitivos de $F A$ (pelo Teorema 1.4.9, no Capítulo 1 , aliado ao fato de que cada $e_{i}$ é a soma de idempotentes primitivos de $F A$, pelo Teorema de Wedderburn - Artin).

Como esses são $(n+1)$ (o número de subgrupos [cíclicos] de $A$ ) idempotentes, temos que serão primitivos sempre que $F A$ tiver exatamente $(n+1)$ componentes 
simples. Como o expoente de $A$ neste caso é $p^{n}$, então pelo visto na seção anterior, isso acontece se, e somente se $q$ e $p^{n}$ são da forma descrita no último corolário da seção anterior.

Portanto, temos o seguinte:

Corolário 2.3.2 Seja F um corpo finito com q elementos, e seja $A$ um grupo cíclico de ordem $p^{n}$. Então, o conjunto de idempotentes descrito no lema anterior é o conjunto de idempotentes primitivos de A se, e somente se um dos seguintes vale:

(i) $p=2$, e ou $n=1$ e q é impar ou $n=2$ e $q \equiv 3(\bmod 4)$;

(ii) p é um primo ímpar e o(q)= $\phi\left(p^{n}\right)$ em $U\left(\mathbb{Z}_{p^{n}}\right)$

O corolário acima é uma generalização do seguinte teorema de Arora e Pruthi (Teorema 3.5 de $[\mathbf{6}]$ ):

Teorema 2.3.3 Seja F um corpo finito com q elementos e A um grupo cíclico com $p^{n}$ elementos, onde p é um primo ímpar tal que o $(q)=\phi\left(p^{n}\right)$ em $U\left(\mathbb{Z}_{p^{n}}\right)$. Seja

$$
A=A_{0} \supset A_{1} \supset \ldots \supset A_{n}=1
$$

a cadeia decrescente de todos os subgrupos de A.

Então o conjunto de idempotentes primitivos de FA é dado por:

$$
\begin{gathered}
e_{0}=\frac{1}{p^{n}}\left(\sum_{a \in A} a\right) ; \\
e_{i}=\widehat{A_{i}}-\widehat{A_{i-1}}, 1 \leq i \leq n .
\end{gathered}
$$

Claramente, estes idempotentes determinam o conjunto dos ideais minimais em $F A$ e, portanto, os códigos cíclicos minimais de comprimento $p^{n}$ sobre $F$.

A partir disso, podemos calcular os idempotentes geradores de ideais minimais no caso de grupos cíclicos de ordem $2 p^{n}$ : 
Teorema 2.3.4 Seja F um corpo finito com q elementos e $G$ um grupo cíclico de ordem $2 p^{n}$, com p primo impar, tal que o $(q)=\phi\left(p^{n}\right)$ em $U\left(\mathbb{Z}_{2 p^{n}}\right)$. Escrevendo $G=C \times A$, onde $A$ é o subgrupo p-Sylow de $G$ e $C=\{1, t\}$ é o seu 2-Sylow. Se $e_{i}, 0 \leq i \leq n$, denotam os primitivos idempotentes de FA então, os idempotentes primitivos de FG são

$$
\begin{aligned}
& \frac{1+t}{2} e_{i}, 0 \leq i \leq n ; \\
& \frac{1-t}{2} e_{i}, 0 \leq i \leq n .
\end{aligned}
$$

Prova: Temos que, como $o(q)$ está em $U\left(\mathbb{Z}_{2 p^{n}}\right)$, então $q$ é ímpar. Sendo assim, aplicando o teorema anterior, temos que os idempotentes primitivos de $F C$ são $(1+t) / 2$ e $(1-t) / 2$. Assim:

$$
F G \simeq F(C \times A)=(F C) A=\left(F \frac{1+t}{2} \oplus F \frac{1-t}{2}\right) A \simeq(F \oplus F) A
$$

Como os idempotentes primitivos de $F C$ correspondem a $(1,0)$ e $(0,1)$ em $(F \oplus F)$ e os idempotentes primitivos de $F A$ são os do teorema anterior, o resultado segue imediatamente.

Depois que nós calcularmos a dimensão dos ideais minimais $I_{i}=(F A)\left(\widehat{A_{i}}-\widehat{A_{i-1}}\right)$ e, portanto, o grau do seu polinômio gerador, calcular o polinômio gerador de $I_{i}$.

\subsection{Códigos Abelianos Minimais}

Queremos estender os resultados da seção anterior para grupos abelianos finitos. Para isso, consideremos primeiro o caso dos p-grupos abelianos finitos.

Seja $A$ um $p$-grupo abeliano finito. Para cada subgrupo $H$ de $A$ tal que $A / H \neq 1$ é cíclico, vamos construir um idempotente de $F A$.

Observemos que, como $A / H$ é um grupo cíclico cuja ordem é uma potência de $p$, então existe apenas um subgrupo $H^{*}$ de $A$ contendo $H$, tal que $\left|H^{*} / H\right|=p$. Vamos definir $e_{H}=\widehat{H}-\widehat{H}^{*} \neq 0$. Temos os seguintes lemas: 
Lema 2.4.1 Seja $R$ um anel e seja $H$ um subgrupo normal de um grupo G. Se $|H|$ é invertível em $R$, então

$$
(R G) \widehat{H} \simeq R(G / H)
$$

Prova: ver Proposição 3.6.7 de [5].

Lema 2.4.2 Seja $G$ um grupo finito e, sejam $H, K$ subgrupos de $G$. Então

$$
\widehat{H} \widehat{K}=\widehat{H K} .
$$

Em particular, se $H \leq K$, então $\widehat{H} \widehat{K}=\widehat{K}$.

Prova: Primeiro, notemos que $h_{1} k_{1}=h_{2} k_{2}$ implica que $h_{2}^{-1} h_{1}=k_{2} k_{1}^{-1} \in H \cap K$, e além disso, $k_{2} k_{1}^{-1}=k k_{1}^{-1}$ se, e somente se $k_{2}=k$ e, portanto, concluímos que

$$
|\{(h, k): h k=\alpha, h \in H, k \in K\}|=|H \cap K|,
$$

para todo $\alpha \in H K$.

Temos que, se $h_{1}, \ldots, h_{n}$, e $k_{1}, \ldots, k_{m}$ são as listas de todos os elementos de $H$ e $K$, respectivamente, então:

$$
\begin{gathered}
\widehat{H} \widehat{K}=\left(\frac{1}{n}\left(h_{1}+\ldots+h_{n}\right)\right)\left(\frac{1}{m}\left(k_{1}+\ldots+k_{m}\right)\right)=\frac{1}{|H||K|} \sum_{h \in H, k \in K} h k= \\
=\frac{|H \cap K|}{|H||K|} \sum_{\alpha \in H K} \alpha=\widehat{H K}
\end{gathered}
$$

como queríamos provar.

Lema 2.4.3 Os elementos $e_{H}$ definidos acima, junto com $e_{A}=\widehat{A}$, formam um conjunto de idempotentes de FA dois a dois ortogonais, cuja soma é 1.

Prova: o fato de que estes elementos são idempotentes segue diretamente do lema anterior. 
Sejam agora $H, K \leq A$ tais que $A / H, A / K$ são cíclicos e não triviais, e sejam $H^{*}, K^{*}$ subgrupos de $A$ contendo $H$ e $K$, respectivamente, tais que $H^{*} / H, K^{*} / K$ são cíclicos de ordem $p$. Vamos considerar primeiro o caso em que $H \subset K$ (propriamente). Neste caso, $H^{*} \subseteq K$ e temos:

$$
e_{H} e_{K}=\left(\widehat{H}-\widehat{H^{*}}\right)\left(\widehat{K}-\widehat{K^{*}}\right)=\widehat{K}-\widehat{K^{*}}-\widehat{K}+\widehat{K^{*}}=0
$$

Agora, se nenhum desses dois subgrupos está contido no outro, então ambos $H$ e $K$ estão contidos propriamente em $H K$, e temos que $H^{*}$ e $K^{*}$ estão contidos em $H K$. Assim, $H^{*} K^{*} \subset H K$, e claro que $H K \subset H^{*} K^{*}$ (pois $H \subset H^{*}, K \subset K^{*}$ ). Então $H^{*} K^{*}=H K$. Agora, como $H K \subset H K^{*} \subset H^{*} K^{*}$, segue que $H K^{*}=H K$ e, analogamente, nós temos $H^{*} K=H K$. Portanto

$$
e_{H} e_{K}=\left(\widehat{H}-\widehat{H^{*}}\right)\left(\widehat{K}-\widehat{K^{*}}\right)=\widehat{H K}-\widehat{H^{*} K}-\widehat{H K^{*}}+\widehat{H^{*} K^{*}}=0
$$

Falta só provar que a soma de todos esses idempotentes é 1. Para cada subgrupo cíclico $C$ de $A$, denotemos por $\mathcal{G}(C)$ o conjunto dos elementos de $C$ que geram este subgrupo; isto é:

$$
\mathcal{G}(C)=\{c \in C: o(c)=|C|\}
$$

Se $\mathcal{C}$ denota a família de todos os subgrupos cíclicos de $A$, então $|A|=\sum_{C \in \mathcal{C}}|\mathcal{G}(C)|$ e, como $A$ é um p-grupo (e também $C$ ), então $|\mathcal{G}(C)|=\phi(|C|)=|C|-|C| / p$.

Seja $\mathcal{S}$ o conjunto de todos os subgrupos $H$ de $A$ tais que o quociente $A / H$ é cíclico e seja $e=\sum_{H \in \mathcal{S}} e_{H}$.

Afirmação: $e=1$.

Para provar tal afirmação, é suficiente provar que $(F A) e=F A$, visto que $e$ é a soma de idempotentes dois a dois ortogonais. E devido a estes idempotentes serem dois a dois ortogonais, temos que

$$
(F A) e=\bigoplus_{H \in \mathcal{S}}(F A) e_{H}
$$


daí, temos

$$
\operatorname{dim}_{F}\left((F A)_{e}\right)=\sum_{H \in \mathcal{S}} \operatorname{dim}_{F}\left((F A)_{e_{H}}\right)
$$

Note que $\widehat{H}=\widehat{H^{*}}+e_{H}$, e que $H^{*} e_{H}=0$ (ou seja, $H^{*}$ e $e_{H}$ são idempotentes e ortogonais entre si), então temos

$$
(F A) \widehat{H}=(F A) \widehat{H^{*}} \oplus(F A) e_{H}
$$

E ainda

$$
\operatorname{dim}_{F}\left((F A) e_{H}\right)=\operatorname{dim}_{F}(F A) \widehat{H}-\operatorname{dim}_{F}(F A) \widehat{H^{*}} .
$$

Segue da equação acima e do Lema 2.12 que

$$
\operatorname{dim}_{F}\left((F A) e_{H}\right)=\operatorname{dim}_{F} F[A / H]-\operatorname{dim}_{F} F\left[A / H^{*}\right]
$$

Temos claramente que:

$$
\begin{gathered}
\operatorname{dim}_{F} F[A / H]=|A / H|, \\
\operatorname{dim}_{F} F\left[A / H^{*}\right]=\left|A / H^{*}\right| .
\end{gathered}
$$

Segundo vimos em 1.4.2, existe uma bijeção $\Phi: \mathcal{C} \rightarrow \mathcal{S}$ tal que, se denotarmos por $C \in \mathcal{C}$ o subgrupo tal que $\Phi(C)=H$, temos $|C|=|A / H|$. Portanto, temos

$$
\begin{gathered}
\operatorname{dim}_{F} F[A / H]=|C|, \\
\operatorname{dim}_{F} F\left[A / H^{*}\right]=\left|A / H^{*}\right|=|A / H| /\left|H^{*} / H\right|=|C| / p,
\end{gathered}
$$

então, pelo teorema acima

$$
\operatorname{dim}_{F}\left((F A) e_{H}\right)=|C|-|C| / p=|\mathcal{G}(C)|,
$$

e portanto

$$
\operatorname{dim}_{F}((F A) e)=\sum_{H \in \mathcal{S}} \operatorname{dim}_{F}\left((F A) e_{H}\right)=\sum_{C \in \mathcal{C}}|\mathcal{G}(C)|=|A|,
$$


e portanto $e=1$, como queríamos.

O seguinte resultado está contido na demonstração acima:

Corolário 2.4.4 Nas condições do lema anterior, temos que

$$
\operatorname{dim}_{F}\left((F A) e_{H}\right)=\operatorname{dim}_{F} F[A / H]-\operatorname{dim}_{F} F\left[A / H^{*}\right]
$$

O seguinte resultado é uma consequência direta do lema que acabamos de provar e do Corolário 2.2.6.

Teorema 2.4.5 Seja p um primo e seja A um p-grupo abeliano finito de expoente $p^{r}$.

Então, o conjunto de idempotentes acima é o conjunto de idempotentes primitivos de FA se, e somente se, um dos seguintes vale:

(i) $p^{r}=2$, e q é impar;

(ii) $p^{r}=4$ e $q \equiv 3(\bmod 4)$;

(iii) p é um primo impar e o(q)= $\phi\left(p^{r}\right)$ em $U\left(\mathbb{Z}_{p^{r}}\right)$.

Prova: se o tal conjunto de idempotentes for de idempotentes primitivos, como sua soma é 1, então estes são todos os idempotentes primitivos, e as hipóteses do Corolário 2.2.6 estarão satisfeitas. Por outro lado, se um dos três itens acima for satisfeito, então o conjunto de idempotentes acima é o conjunto de idempotentes primitivos de $F A$, pelo Corolário 2.2.6.

Também, temos o seguinte:

Teorema 2.4.6 Seja p um primo impar e seja A um grupo abeliano de expoente $2 p^{r}$, e seja $F$ um corpo finito com $q$ elementos, onde o $(q)=\phi\left(2 p^{r}\right)$ em $U\left(\mathbb{Z}_{2 p^{r}}\right)$ (em particular, temos que q é impar). Escrevamos $A=E \times B$, onde $E$ é um 2-grupo abeliano elementar e $B$ é um p-grupo. Então os idempotentes primitivos de $F$ A são todos os produtos da forma e.f, onde e é um idempotente primitivo de $F E$ e f é um idempotente primitivo de FB. 
Prova: escrevendo $E=\left\langle a_{1}\right\rangle \times \ldots \times\left\langle a_{n}\right\rangle$, um produto de grupos cíclicos, temos que

$$
F A \simeq F(E \times B) \simeq(F E) B
$$

$\mathrm{e}$

$(F E) \simeq F\left(\left\langle a_{1}\right\rangle \times \ldots \times\left\langle a_{n}\right\rangle\right) \simeq\left(F\left\langle a_{1}\right\rangle\right)\left(\left\langle a_{2}\right\rangle \times \ldots \times\left\langle a_{n}\right\rangle\right) \simeq(F \oplus F)\left(\left\langle a_{2}\right\rangle \times \ldots \times\left\langle a_{n}\right\rangle\right)$

Continuando indutivamente o processo acima, temos que $F E \simeq F^{2^{n}}$, e imediatamente temos o desejado.

Temos ainda que, com as hipóteses do teorema acima, os idempotentes primitivos de $F B$ são dados no teorema anterior.

Escrevendo $E=\left\langle a_{1}\right\rangle \times \ldots \times\left\langle a_{n}\right\rangle$, um produto de grupos cíclicos, então os idempotentes primitivos de $F E$ são todos os produtos do tipo $e=e_{1} \ldots e_{n}$, onde

$$
\begin{gathered}
e_{i}=\frac{1+a_{i}}{2}, \text { ou } \\
e_{i}=\frac{1-a_{i}}{2}, 1 \leq i \leq n,
\end{gathered}
$$

pois temos, pela demonstração do teorema acima, que $F E \simeq F^{2^{n}}$ e, portanto, $F E$ tem exatamente $2^{n}$ idempotentes primitivos, e estes elementos descritos acima são $2^{n}$ idempotentes todos ortogonais entre si e somam 1 e, portanto, são os idempotentes primitivos de $F E$.

Sendo assim, temos uma descrição completa dos idempotentes primitivos de $F A$, nas hipóteses do teorema anterior.

Vale notar que, pelo Corolário 2.2.6, já temos os únicos casos em que os idempotentes primitivos de álgebras de grupos abelianos finitos sobre corpos finitos podem ser calculados desta forma. 


\subsection{Dimensão e Distância Mínima}

Nesta seção, calcularemos a dimensão de certos códigos abelianos minimais sobre $F$ e a distância mínima dos mesmos. Para isso precisaremos das seguintes definições:

Definição 2.5.1 Dado um ideal I de FG, onde $G$ é um grupo finito, sejam a = $\sum_{g \in G} a_{g} g, b=\sum_{g \in G} b_{g} g$ elementos de $G$. Então definimos a distância entre a e $b$ como sendo

$$
d(a, b)=\left|\left\{g \in G: a_{g} \neq b_{g}, g \in G\right\}\right|
$$

Definimos a distância mínima do ideal I como sendo

$$
l(I)=\min \{d(a, b): a, b \in I, a \neq b\}
$$

Definimos o peso de um elemento a $\in F G$ como sendo

$$
w(a)=\left|\left\{g \in G: a_{g} \neq 0\right\}\right|
$$

note que $w(a)=d(a, 0)$.

E definimos o peso de um ideal I de FG como sendo

$$
w(I)=\min \{w(a): a \in I, a \neq 0\}
$$

Obs.: temos que $d(a, b)=w(a-b)$. Se $w(I)=c$, então existe $0 \neq a \in I$ tal que $w(a)=c=d(a, 0)$. Logo $c \in\{d(a, b): a \neq b\}$ e, portanto, $l(I) \leq w(I)$. E como $d(a, b)=w(a-b)$, para todos $a, b \in F G$, temos $w(I) \leq l(I)$. Então $w(I)=l(I)$, para todo ideal $I$ de $F G$.

Suponha que $|A|=2^{m} p^{n}$, onde $p$ é um primo ímpar e $m \geq 0$. Como fizemos antes, escrevamos $A=E \times B$, onde $E$ é um 2-grupo abeliano elementar (eventualmente trivial) e $B$ um p-grupo.

Como foi notado no fim da seção anterior, os idempotentes primitivos de $F E$ são todos os produtos da forma $e_{E}=e_{1} \ldots e_{m}$, onde

$$
e_{i}=\frac{1+a_{i}}{2}, \text { ou } e_{i}=\frac{1-a_{i}}{2}, 1 \leq i \leq m
$$


e os idempotentes primitivos de $F A$ são todos os produtos da forma $e_{E} e_{B}$, onde $e_{E}$ é um idempotente primitivo de $F E$ e $e_{B}$ é um idempotente primitivo de $F B$.

Para um idempotente primitivo $e_{E}$ de $F E$ fixo e um elemento $y \in E$, podemos escrever $y=a_{1}^{\epsilon_{1}} \ldots a_{m}^{\epsilon_{m}}$, onde $\epsilon_{i}=0$ ou 1 . Temos

$$
y e_{E}=a_{1}^{\epsilon_{1}}\left(\frac{1 \pm a_{1}}{2}\right) \ldots a_{m}^{\epsilon_{m}}\left(\frac{1 \pm a_{m}}{2}\right)= \pm e_{E}=(-1)^{\epsilon_{y}} e_{E},
$$

onde $\epsilon_{y}=0$ ou 1 .

Considere primeiro os idempotentes da forma $e_{E} \widehat{B}$. Um elemento de $(F A) \cdot e_{e} \widehat{B}$ é da forma $\gamma \cdot e_{E} \widehat{B}$, tal que $\gamma$ pode ser escrito $\gamma=\sum_{y \in E, b \in B} x_{y b} y b$, onde cada $x_{y b}$ é um elemento do corpo $F$; e então nós temos que

$$
\gamma \cdot e_{E} \widehat{B}=\sum_{y \in E, b \in B} x_{y b} y e_{E} \cdot b \widehat{B} \stackrel{(2.2)}{=}\left(\sum_{y \in E, b \in B} x_{y b}(-1)^{\epsilon_{y}}\right) e_{E} \widehat{B}
$$

Como $\left(\sum_{y \in E, b \in B} x_{y b}(-1)^{\epsilon_{y}}\right) \in F$, temos que a dimensão do ideal $I=(F A) \cdot e_{E} \widehat{B}$ é 1 , e que seu peso é $w(I)=|A|\left(\right.$ pois $\operatorname{supp}\left(e_{E} \widehat{B}\right)=A$, e para cada elemento do ideal, todos os coeficientes são iguais).

Agora, consideremos os idempotentes do tipo $e=e_{E} e_{H}$, com $e_{E} \in F E$, como acima, e $e_{H}=\widehat{H}-\widehat{H^{*}}$, onde $H$ é um subgrupo de $B$ tal que $B / H$ é cíclico de ordem, digamos, $p^{i}$, e $H^{*}$ é o único subgrupo de $B$ que contém $H$ tal que $\left[H^{*} / H\right]=p$. Seja $I_{e}=(F A) e$.

Seja $b \in B$ um elemento tal que $B=\langle b, H\rangle$ (tome $b$ um representante da co-classe do gerador do grupo cíclico $B / H)$. Assim, temos também que $H^{*}=\left\langle b^{p^{i-1}}, H\right\rangle$.

Como $b^{p^{i-1}} \in H^{*}$, temos que $\left(1-b^{p^{i-1}}\right) \widehat{H^{*}}=0$.

Então segue que

$$
\left(1-b^{p^{i-1}}\right) e_{E} \widehat{H}=\left(1-b^{p^{i-1}}\right) e_{E}\left(\widehat{H^{*}}+e_{H}\right)=\left(1-b^{p^{i-1}}\right) e_{E} e_{H} \in I_{E}
$$

Como $b^{p^{i-1}} \notin H$, então $\operatorname{supp}\left(\left(1-b^{p^{i-1}}\right) \widehat{H}\right)$ é a união disjunta $H \cup b^{p^{i-1}} H$, e o peso do elemento acima é $w\left(\left(1-b^{p^{i-1}}\right) e_{E} \widehat{H}\right)=2|E||H|$, de tal forma que a distância 
mínima do ideal $I_{e}$ é $l\left(I_{e}\right) \leq 2^{m+1}|H|$.

Como $B$ é igual à união disjunta $B=H \cup b H \cup \ldots \cup b^{p^{i}-1} H$, então nós temos que $A=E \times B$ é a união disjunta $A=E \times H \cup \ldots \cup b^{p^{i}-1}(E \times H)$, de tal forma que um elemento arbitrário de $F A$ pode ser escrito na forma $\alpha=\sum_{j=0}^{p^{i}-1} \alpha_{j} b^{j}$, com $\alpha_{j} \in F[E \times H]$.

Pela fórmula (2.2), e utilizando o fato de que, se $h \in H$, vale que $h \widehat{H}=\widehat{H}$, temos que cada produto $\alpha_{j} e_{E} e_{H}$ é da forma $\alpha j e_{E} e_{H}=k_{j} e_{E} e_{H}$, onde $k_{j} \in F, 0 \leq j \leq p^{i}-1$ (O mesmo vale pondo $\widehat{H}$ no lugar de $\left.e_{H}: \alpha j e_{E} \widehat{H}=k_{j} e_{E} \widehat{H}\right)\left(^{*}\right)$.

Como $e_{E} e_{H} \cdot e_{E} \widehat{H}=e_{E} e_{H}$, temos que $(F A) \cdot e_{E} e_{H} \subset(F A) \cdot e_{E} \widehat{H}$, e esta inclusão é estrita, pois se existisse $\beta \in F A$ tal que $\beta e_{E} e_{H}=e_{E} \widehat{H}$, multiplicando por $e_{H}$ dos dois lados, concluiríamos que $e_{E} \widehat{H}=e_{E} e_{H}$, o que não pode ser verdade, pois $b^{p^{i-1}}$ está no suporte do membro da direita, mas não no suporte do membro da esquerda desta última igualdade. Portanto, um elemento $0 \neq \gamma \in(F A) \cdot e_{E} e_{H}=I_{e}$ pode ser escrito na forma

$$
\gamma=\alpha e_{E} \widehat{H} \stackrel{(*)}{=}\left(k_{0}+k_{1} b+\ldots+k_{p^{i}-1} b^{p^{i}-1}\right) e_{E} \widehat{H}
$$

Como $\gamma \neq 0$, temos que pelo menos um dos coeficientes $k_{j} \neq 0$. Se $\gamma=k_{j} b^{j} e_{E} \widehat{H}$, teríamos que $e_{E} \widehat{H} \in(F A) \cdot e_{E} e_{H}$. Mas isso não pode acontecer, pois a inclusão $(F A) \cdot e_{E} e_{H} \subset(F A) \cdot e_{E} \widehat{H}$ é estrita. Então, pelo menos dois $k_{j}$ 's diferentes são diferentes de 0 . Como $\gamma \neq 0$ foi pego arbitrário em $I_{e}$, temos que $l\left(I_{e}\right) \geq 2^{m+1}|H|$. Como a inclusão oposta nós já tínhamos concluído, temos portanto que

$$
l\left(I_{e}\right)=2^{m+1}|H|
$$

e calculamos a distância mínima de todos os ideais minimais.

Finalmente, vamos calcular a dimensão dos códigos abelianos minimais; isto é, a dimensão dos ideais da forma $F$ A.e, onde e é um idempotente primitivo de $F A$.

Teorema 2.5.2 Seja $e=e_{E} e_{H}$ um idempotente primitivo de $F A$, conforme as definições acima. Temos que:

$$
\operatorname{dim}(F A . e)=\phi\left(p^{i}\right)
$$




$$
\operatorname{dim}\left(F A \cdot e_{E} \widehat{B}\right)=1
$$

Prova:Temos

$$
F A . e_{E} e_{H}=F[E \times B] . e_{E} e_{H}=((F E) B) \cdot e_{E} e_{H}=\left(F E . e_{E}\right) B \cdot e_{H} .
$$

Pela fórmula (2.2), temos que $(F E) \cdot e_{E} \simeq F$, para todo idempotente primitivo $e_{E}$ de $F E$. E segue da fórmula acima que

$$
F A . e_{E} e_{H} \simeq F B \cdot e_{H}
$$

e do Corolário 2.4.4, temos que

$$
\operatorname{dim}\left[F A \cdot e_{E} e_{H}\right]=\phi\left(p^{i}\right) .
$$

Agora vamos ao caso em que $e_{H}=\widehat{B}$. Como já vimos ao calcular a distância mínima do ideal $(F A) \cdot e_{E} \widehat{B}$, todo elemento deste ideal é um múltiplo por um elemento de $F$ do elemento $e_{E} \widehat{B}$. Assim, temos

$$
\operatorname{dim}\left[F A \cdot e_{E} \widehat{B}\right]=\operatorname{dim}[F B \cdot \widehat{B}]=1
$$

\subsection{Polinômio Gerador de Códigos Cíclicos Minimais de Ta- manho $p^{n}$}

Agora, para completar, vamos encontrar qual é o polinômio gerador dos códigos cíclicos $I_{i}=(F A)\left(\widehat{A_{i}}-\widehat{A_{i-1}}\right)\left(\right.$ como descrito no Teorema 2.10), e $I_{0}=(F A)(\widehat{A})$, onde $A$ é cíclico de ordem $p^{n}$, e $F$ é corpo finito com $q$ elementos, e $(q,|A|)=1$.

Começaremos com o caso $i>0$ :

Pela Proposição 1.4.21, temos que, se $e_{i}(x)$ corresponde ao idempotente primitivo $e_{i}$, então o polinômio gerador $g_{i}(x)$ do ideal $I=(F A) e_{i}$ satisfaz: 


$$
g_{i}(X)=m d c\left(e_{i}(X), X^{p^{n}}-1\right) .
$$

Então, basta escolher $e_{i}(x)$ tal que $e_{i}(a)=e_{i}$, onde $a$ é gerador de $A$. Então vamos escolher

$$
e_{i}(x)=\frac{1}{p^{n-i}} \sum_{j=0}^{p^{n-i}-1} X^{j p^{i}}-\frac{1}{p^{n-i+1}} \sum_{j=0}^{p^{n-i+1}-1} X^{j p^{i-1}}
$$

Vamos simplificar a expressão acima:

$$
\begin{aligned}
e_{i}(X) & =\frac{1}{p^{n-i}} \sum_{j=0}^{p^{n-i}-1} X^{j p^{i}}-\frac{1}{p^{n-i+1}} \sum_{j=0}^{p^{n-i+1}-1} X^{j p^{i-1}} \\
= & \frac{1}{p^{n-i+1}}\left[p \sum_{j=0}^{p^{n-i}-1} X^{j p^{i}}-\sum_{j=0}^{p^{n-i+1}-1} X^{j p^{i-1}}\right]
\end{aligned}
$$

Note que

$$
\begin{gathered}
\left(\sum_{j=1}^{p-1} X^{j p^{i-1}}\right)\left(\sum_{j=0}^{p^{n-i}-1} X^{j p^{i}}\right)=\sum_{k=1}^{p-1} \sum_{j=0}^{p^{n-i}-1} X^{k p^{i-1}+j p p^{i-1}}= \\
=\sum_{k=1}^{p-1} \sum_{j=0}^{p^{n-i}-1} X^{(k+j p) p^{i-1}}=\sum_{j=1}^{p^{n-i+1}-1} X^{j p^{i-1}} .
\end{gathered}
$$

Portanto, temos

$$
\begin{gathered}
=\frac{1}{p^{n-i+1}}\left[p \sum_{j=0}^{p^{n-i}-1} X^{j p^{i}}-\sum_{j=0}^{p^{n-i+1}-1} X^{j p^{i-1}}\right]= \\
=\frac{1}{p^{n-i+1}}\left[(p-1)\left(\sum_{j=0}^{p^{n-i}-1} X^{j p^{i}}\right)-\left(\sum_{j=1}^{p-1} X^{j p^{i-1}}\right)\left(\sum_{j=0}^{p^{n-i}-1} X^{j p^{i}}\right)\right] \\
=\frac{1}{p^{n-i+1}}\left(p-\sum_{j=0}^{p-1} X^{j p^{i-1}}\right)\left(\sum_{j=0}^{p^{n-i}-1} X^{j p^{i}}\right)
\end{gathered}
$$

Também: 


$$
X^{p^{n}}-1=\left(X^{p^{i}}-1\right) \sum_{j=0}^{p^{n-i}-1} X^{j p^{i}}=\left(X^{p^{i-1}}-1\right)\left(\sum_{j=0}^{(p-1) p^{i-1}} X^{j p^{i-1}}\right)\left(\sum_{j=0}^{p^{n-i}-1} X^{j p^{i}}\right) .
$$

Notando que $X^{j p^{i-1}}=\left(X^{p^{i-1}}\right)^{j}$, concluímos imediatamente toda raiz de $\left(X^{p^{i-1}}-\right.$ 1) em um fecho algébrico de $F$ é também uma raiz de

$$
p-\sum_{j=0}^{p-1} X^{j p^{i-1}}
$$

e, portanto, $\left(X^{p^{i-1}}-1\right)$ divide $p-\sum_{j=0}^{p-1} X^{j p^{i-1}}$.

Como já sabemos que a dimensão de $I_{i}=\phi\left(p^{i}\right)=p^{i}-p^{i-1}$ é igual a $p^{n}-$ $\operatorname{deg}\left(g_{i}(X)\right)$ (pela observação logo após a Definição 1.4.18), temos que

$$
g_{i}(X)=\left(X^{p^{i-1}}-1\right)\left(\sum_{j=0}^{p^{n-i}-1} X^{j p^{i}}\right)
$$

E assim concluímos o caso $i>0$.

Agora vamos analisar o caso $i=0$ :

Como fizemos na escolha dos $e_{i}$ 's acima, podemos escolher $e_{0}(X)=\sum_{i=0}^{p^{n}-1} X^{i}$, que tem grau $p^{n}-1$. Como $g_{0}(X)=m d c\left(e_{0}(X), X^{p^{n}}-1\right)$, e além disso $\left(X^{p^{n}}-1\right)=$ $e_{0}(X)(X-1)$, temos que $g_{0}(X)=e_{0}(X)$.

Assim, concluímos este capítulo. 


\section{Capítulo 3}

\section{Idempotentes Centrais Primitivos em}

\section{Álgebras de grupo Racionais de Grupos Nilpotentes Finitos}

O objetivo deste capítulo é descrever quais são os idempotentes centrais primitivos de uma álgebra de grupo racional de um grupo nilpotente finito, sem fazer uso da tabela de caracteres do grupo G, e utilizando para isso o artigo Central idempotents in the rational group algebra of a finite nilpotent group, de E., Jespers, G., Leal e A., Paques. Descreveremos, em primeiro lugar, os idempotentes primitivos de $\mathbb{Q} G$ quando $G$ é um grupo abeliano finito.

Precisaremos ainda de mais alguns pré-requisitos, e começaremos este capítulo por eles.

\subsection{Pré-requisitos}

Antes de partirmos para o problema proposto acima, vamos considerar alguns dos requisitos de que precisamos. Começaremos com um pouco de representações de grupos:

Definição 3.1.1 Seja $G$ um grupo e $V$ um $\mathbb{C}$ - espaço vetorial de dimensão finita. Uma representação de $G$ (sobre $\mathbb{C}$ ), com espaço de representação $V$, é um homomorfismo de grupos 


$$
\begin{gathered}
T: G \rightarrow L(V) \\
T(g) \mapsto T_{g},
\end{gathered}
$$

onde $L(V)$ é o grupo de operadores lineares bijetores de $V$ (que pode ser identificado com o grupo de matrizes quadradas de tamanho $\operatorname{dim}_{\mathbb{C}} V$ e determinante não nula).

Uma tal representação é dita fiel se T for injetora.

Uma representação é dita irredutivel se $V \neq\{0\}$ e os únicos subespaços de $V$ invariantes sob todos os $T_{g}$ 's são os triviais: $\{0\}$ e $V$.

Temos o seguinte resultado:

Lema 3.1.2 (Schür) Seja $G$ um grupo e $T: G \rightarrow M_{n}(\mathbb{C})$ uma representação irredutivel de $G$. Então, se $M \in M_{n}(\mathbb{C})$ comuta com cada elemento $T_{g}$ da imagem de $T$, então $M \in Z\left(M_{n}(\mathbb{C})\right)$, ou seja, $M$ é um múltiplo da matriz identidade.

Prova: seja $M \in M_{n}(\mathbb{C})$ matriz que comuta com cada elemento da imagem de $T$. Como $\mathbb{C}$ é algebricamente fechado, então existe um autovalor $\lambda \in \mathbb{C}$ da matriz $M$. Seja $V_{\lambda}$ o auto-espaço correspondente. Vamos provar que $V_{\lambda}$ é invariante sobre todo $T_{g} \in T(G)$. Seja $v \in V_{\lambda}$ :

$$
\begin{gathered}
M T_{g}(v)=T_{g} M(v)=T_{g}(\lambda v)=\lambda T_{g}(v) \\
\Rightarrow T_{g}(v) \in V_{\lambda} .
\end{gathered}
$$

Portanto, temos que, como $V_{\lambda} \neq\{0\}$ e a representação é irredutível, então $V_{\lambda}=V$ e, portanto, $M=\lambda I$.

Continuaremos denotando o centro de um grupo $G$ por $Z(G)$.

Temos o seguinte corolário:

Corolário 3.1.3 Se G é um grupo finito que tem representação irredutível e fiel, então $Z(G)$ é um grupo cíclico. 
Prova: Seja $T$ uma tal representação. Pelo lema anterior, temos que cada elemento de $T(Z(G))$ é um escalar multiplicado pela matriz identidade. Portanto, temos que, naturalmente, $T(Z(G))$ é isomorfo a um subgrupo finito do grupo multiplicativo $\mathbb{C}^{*}$. Como todo subgrupo finito do grupo multiplicativo de um corpo é cíclico, temos que $T(Z(G))$ é cíclico. Como a representação é fiel, temos que $Z(G) \simeq T(Z(G))$, e temos o resultado que desejamos.

Tendo os resultados desta seção em mente, vamos agora às partes principais do capítulo.

\subsection{Quando $G$ é Grupo Abeliano Finito}

De agora em diante, $G$ sempre denotará um grupo finito.

Defina, para e um idempotente central primitivo de $\mathbb{K} G$, o subgrupo

$$
G_{e}=\{g \in G: g e=e\}
$$

Temos que $G_{e}$ é subgrupo normal de $G$, pois se $h e=e\left(h \in G_{e}\right)$, então para $g \in G$ temos

$$
\left(g h g^{-1}\right) e=(g h)\left(g^{-1} e\right)=(g h)\left(e g^{-1}\right)=g(h e) g^{-1}=g e g^{-1}=g g^{-1} e=e
$$

Como temos o resultado ([5], Lema 3.6.6) de que, para $H \leq G, \widehat{H}$ é sempre idempotente em $\mathbb{Q} G$, e é central se $H$ é normal, então temos que $\widehat{G_{e}}$ é idempotente central de $\mathbb{Q} G$.

Além disso,

$$
e \widehat{G_{e}}=e \frac{1}{\left|G_{e}\right|} \sum_{g \in G_{e}} g=\frac{1}{\left|G_{e}\right|} \sum_{g \in G_{e}} e g=\frac{1}{\left|G_{e}\right|} \sum_{g \in G_{e}} e=e .
$$

Portanto, temos que e é um idempotente central primitivo de $(\mathbb{Q} G) \widehat{G_{e}} \simeq \mathbb{Q}\left(G / G_{e}\right)$, então é é idempotente central primitivo de $\mathbb{Q}\left(G / G_{e}\right)$. 
Se $G$ for não trivial, denotemos por $\mathcal{M}(G)$ o conjunto de todos os subgrupos normais minimais de $G$. E vamos definir

$$
\varepsilon(G)=\prod_{M \in \mathcal{M}(G)}(1-\widehat{M}) .
$$

E definimos $\varepsilon(\{1\})=1$.

Seja $N$ um subgrupo normal de $G$ e seja $M$ um subgrupo de $G$ que contém $N$. Então já sabemos que $\widehat{N} \widehat{M}=\widehat{M}$ (pelo Lema 2.4.2). Denotemos por $\bar{M}$ o grupo $M / N$. Se $N \neq G$ definimos

$$
\varepsilon(G, N)=\prod_{\bar{M} \in \mathcal{M}(G / N)}(\widehat{N}-\widehat{M})=\prod_{\bar{M} \in \mathcal{M}(G / N)}(\widehat{N}-\widehat{N} \widehat{M})=\widehat{N} \prod_{\bar{M} \in \mathcal{M}(G / N)}(1-\widehat{M})
$$

E definimos $\varepsilon(G, G)=\widehat{G}$. Desta forma, temos que a pré-imagem de $\varepsilon(G / N)$ em $(\mathbb{Q} G) \widehat{N}$ é $\varepsilon(G, N)$. Obs.: aqui estamos utilizando o isomorfismo $\left((\mathbb{Q} G) \widehat{G_{e}}\right) \simeq \mathbb{Q}\left(G / G_{e}\right)$, encontrado na demonstração da Proposição 3.6.7 de [5], que identifica $G / G_{e}$ e $G \widehat{G_{e}}$ como grupos, pelo morfismo $\phi: G \rightarrow G \widehat{G_{e}}$, dado por $\phi(g)=g \widehat{G_{e}}$, que é um epimorfismo de núcleo $G_{e}$.

Temos o seguinte lema:

Lema 3.2.1 Se e é um idempotente central primitivo de $\mathbb{Q} G$ e $N$ é um subgrupo normal de $G$, então e $\widehat{N}=e$ se, e somente se, $N \subset G_{e}$.

Prova: Se $N \subset G_{e}$, temos:

$$
e \widehat{N}=e \frac{1}{|N|} \sum_{g \in N} g=\frac{1}{|N|} \sum_{g \in N} e g=\frac{1}{|N|} \sum_{g \in N} e=e .
$$

Por outro lado, se $e \widehat{N}=e$, seja $n \in N$. Temos

$$
e n=(e \widehat{N}) n=e(\widehat{N} n)=e \widehat{N}=e,
$$


portanto $n \in G_{e}$ e, logo, $N \subset G_{e}$.

Observemos que, se $f$ é um idempotente central, como $e=e f+e(1-f)$, e cada um dos termos é idempotente e central, e são ortogonais entre si (isto é, $(e f)(e(1-f))=$ $0)$, como $e$ é idempotente central primitivo segue que ef $=e$ ou ef $=0$. Em particular, se $N$ é como no lema anterior, então $e \widehat{N}=e$ ou 0 .

Há outros lemas de que precisaremos:

Lema 3.2.2 Se e é um idempotente central primitivo de $\mathbb{Q} G$ e $G_{e}=\{1\}$, então $G$ tem representação irredutível fiel.

Prova: Seja e como no enunciado. Temos que $e$ um idempotente central (não necessariamente primitivo) de $\mathbb{C} G$. Assim sendo, existem idempotentes centrais primitivos $e_{1}, \ldots, e_{m}$ de $\mathbb{C} G$ distintos tais que $e=e_{1}+\ldots+e_{m}$. Como $\mathbb{C} G e_{i}$ é um módulo semissimples, então existem ideais à esquerda minimais $I_{i, j}$ de $\mathbb{C} G e_{i}$ tais que

$$
\mathbb{C} G e_{i}=I_{i, 1} \oplus \ldots \oplus I_{i, n_{i}} .
$$

Podemos escolher os $I_{i, j}$ como sendo matrizes coluna, (conforme vimos no Teorema 1.4.6 (iv)).

Temos homomorfismos $T_{i, k}$ do tipo:

$$
\begin{gathered}
T_{i, k}: G \rightarrow L\left(I_{i, k}\right) \\
g \mapsto T_{g}, 1 \leq k \leq n_{i}, 1 \leq i \leq m,
\end{gathered}
$$

onde $T_{g}(x)=g x$. Como $x$ pode ser escrito como sendo $x=e_{i} y(y \in \mathbb{C} G)$, temos que $T_{g}(x)=T_{g}\left(e_{i} y\right)=g e_{i} y=\left(g e_{i}\right)\left(e_{i} y\right)$, então de fato $T_{g}$ está definida em $I_{i, k}$. Além disso, temos que cada $T_{g}$ é injetora (e, portanto, bijetora), pois $T_{g} x=T_{g} z$ se, e somente se $g x=g z$, e este último acontece se, e somente se $x=z$. Portanto as funções do tipo $T_{i, k}$ acima são representações de $G$. 
Além disso, cada representação $T_{i, k}$ é irredutível: suponha que existe um $\mathbb{C}$ subespaço $V$ de $I_{i, k}$ invariante sob todos os elementos da imagem de $T_{i, k}$. Isto implica que $(\mathbb{C} G) V \subset V$ e, em particular, $V$ é um ideal à esquerda de $\mathbb{C} G e_{i}$. Como $I_{i, k}$ é ideal à esquerda minimal, então $V=\{0\}$ ou $V=I_{i, k}$.

Além disso temos, pela escolha dos $I_{i, k}$ 's como sendo matrizes coluna que, para cada par $(i, k), T_{i, k}$ é fiel se, e somente se $T_{i, j}$ for fiel, para todo $j$. E este último acontece se, e somente se $G_{e_{i}}=\{1\}$. Portanto, para provar o nosso resultado, temos de provar que $G_{e_{i}}=\{1\}$, para algum $i$.

Como já vimos, temos que cada $\widehat{G_{e_{i}}}$ é um idempotente central em $\mathbb{Q} G$ (pois $G_{e_{i}}$ é normal em $G$ ) e, assim, $e \widehat{G_{e_{i}}}=0$ ou $e \widehat{G_{e_{i}}}=e$.

Temos

$$
e \widehat{G_{e_{i}}}=\left(e_{1}+\ldots+e_{m}\right) \widehat{G_{e_{i}}}=\widehat{G_{e_{i}}} e_{1}+\ldots+e_{i}+\ldots \widehat{G_{e_{i}}} e_{m} \neq 0
$$

Portanto, $e \widehat{G_{e_{i}}}=e$ e, pelo Lema 3.2.1, $G_{e_{i}} \subset G_{e}=\{1\}$ e, portanto, $G_{e_{i}}=\{1\}$, como queríamos.

O resultado do lema acima é, na verdade, uma equivalência. Apesar de que não precisaremos da afirmação recíproca, vamos prová-la para fins de completude:

Lema 3.2.3 Se e é um idempotente central primitivo de $\mathbb{Q} G$, então $G_{e}=\{1\}$ se, e somente se G tem representação fiel irredutivel.

Prova: O lema anterior nos diz que se $G_{e}=\{1\}$, então $G$ tem representação fiel irredutível.

Suponha agora que e é um idempotente central primitivo de $\mathbb{Q} G$ e $G$ tem representação fiel irredutível. Vamos utilizar as mesmas notações da prova do teorema anterior:

Pelo Teorema 1.6, temos que todos os $\mathbb{Q} G$-módulos irredutíveis são isomorfos aos $I_{j, k}$ da prova do lema anterior. Como $G$ tem representação fiel e irredutível, temos, pelas Proposições 4.2.1 e 4.2.2(ii) de [5] (que junto com o Teorema 1.4.6 dizem que 
toda representação irredutível de $G$ é equivalente a uma das representações $T_{i, j}$ ) e pela demonstração do lema anterior que $G_{e_{i}}=\{1\}$, para algum $i$. Portanto, temos que $G_{e}=\{1\}$, como queríamos.

E temos o seguinte lema, como consequência:

Lema 3.2.4 Seja G um grupo finito e seja e um idempotente central primitivo de $\mathbb{Q} G$. Então $G_{e}=\{1\}$ se, e somente se $\varepsilon(G) e=e$. Em particular, $\varepsilon(G) \neq 0$ se, $e$ somente se G tem uma representação fiel irredutivel.

Prova: Suponha que $G_{e}=\{1\}$. Se $M \in \mathcal{M}(G)$, então $\widehat{M}$ é idempotente central e, desta forma, temos que $e \widehat{M}=e$ ou 0 mas, como $G_{e}=\{1\}$, temos, pelo Lema 3.2.1, que $e \widehat{M}=0$, para todo $M \in \mathcal{M}(G)$. Como $G$ é finito, temos que $\mathcal{M}(G)=M_{1}, \ldots, M_{n}$ um conjunto finito. E temos

$$
\begin{gathered}
\varepsilon(G) e=\prod_{M \in \mathcal{M}(G)}(1-\widehat{M}) e=\left(1-\widehat{M}_{1}\right) \ldots\left(1-\widehat{M}_{n}\right) e \\
=\left(1-\widehat{M}_{1}\right) \ldots\left(1-\widehat{M_{n-1}}\right)(e-0)=\left(1-\widehat{M}_{1}\right) \ldots\left(1-\widehat{M_{n-1}}\right) e .
\end{gathered}
$$

Repetindo o passo acima mais $n-1$ vezes, temos que o produto acima é $e$.

Por outro lado, suponha que $\varepsilon(G) e=e$. Se $G_{e} \neq\{1\}$, então existe $M \in \mathcal{M}(G)$ subgrupo não trivial de $G_{e}$ e normal em $G$ e, portanto, $\varepsilon(G) e=0 \neq 1$, contradizendo a hipótese.

Em particular: suponha $\varepsilon(G) \neq 0$. Como $\varepsilon(G)$ é idempotente central, então existem idempotentes centrais primitivos $e_{1}, \ldots, e_{k}$ tais que $\varepsilon(G)=e_{1}+\ldots+e_{k}$ e, portanto, $\varepsilon(G) e_{1}=e_{1}$. Das partes anteriores, temos que $G_{e_{1}}=\{1\}$. Logo, $\varepsilon(G) \neq 0$ se, e somente se, existe idempotente central primitivo $e_{1}$ tal que $G_{e_{1}}=\{1\}$. Assim, o resultado segue pelo Lema 3.2.3.

O $i$-ésimo centro de $G$ ainda denotaremos por $Z_{i}(G)$. O centro denotaremos apenas por $Z(G)$. Lembremos aqui o conhecido fato de que, num grupo nilpotente, 
todo subgrupo normal intersecta o centro de forma não trivial (Teorema 1.4.29) e, portanto, todo subgrupo normal minimal de um grupo nilpotente é central (isto é, está contido no centro); em particular, todo subgrupo normal minimal de um grupo nilpotente é um grupo cíclico de ordem prima.

Temos, portanto, o seguinte resultado:

Lema 3.2.5 Seja $G$ um grupo finito. $S e \varepsilon(G) \neq 0$, então $Z(G)$ é cíclico. A recíproca vale se, além diso, $G$ for um grupo nilpotente.

Prova: Se $\varepsilon(G) \neq 0$, pelo Lema 3.2.4 temos que $G$ tem representação irredutível fiel e, pelo Corolário 3.1.3, temos que $Z(G)$ é cíclico.

Por outro lado, suponha $G$ nilpotente e $Z(G)$ cíclico. Como observado anteriormente, todo subgrupo normal minimal de $G$ é cíclico de ordem $p$. Pela unicidade dos subgrupos de ordem fixa de um grupo cíclico, e do fato de que, em um grupo nilpotente, todo subgrupo normal não trivial intersecta o centro não-trivialmente, temos que cada subgrupo normal minimal de $G$ tem ordem prima, e todos têm ordens distintas e estão contidos no centro de $G$. Assim, pelo teorema da decomposição de grupos abelianos finitos, temos que $Z(G)$ pode ser escrito como o produto direto interno:

$$
Z(G)=P_{1} \ldots P_{k}
$$

onde cada $P_{i}$ é um $p_{i}$-Subgrupo $\left(p_{i}\right.$ primo), com $p_{i} \neq p_{j}$, se $i \neq j$. Temos que cada subgrupo normal minimal de $G$ é o subgrupo (cíclico) $C_{p_{i}}=\left\langle a_{i}\right\rangle$ de ordem $p_{i}$ contido em $P_{i}$, para algum $i$.

$$
\varepsilon(G)=\prod_{i=1}^{k}\left(1-\widehat{\left\langle a_{i}\right\rangle}\right)
$$

Assim sendo, como o produto (3.1) é direto, temos, pelo Lema 2.4.2, distribuindo o produto acima, que o coeficiente de $g=a_{1} \ldots a_{k}$ em $\varepsilon(G)$ é $\frac{1}{|C|} \neq 0$, e temos, portanto, que $\varepsilon(G) \neq 0$. 
Como aplicação dos lemas anteriores, vamos descrever quais são os idempotentes centrais primitivos da álgebra de grupo racional de um grupo abeliano finito. Mas antes, vamos precisar de um lema:

Lema 3.2.6 Seja $A=G$ um grupo cíclico finito. Então $\varepsilon(A)$ é um idempotente primitivo de $\mathbb{Q} A$.

Prova: seja $a \in A$ tal que $A=\langle a\rangle$. Temos $\varepsilon(A)=\prod_{M \in \mathcal{M}(A)}(1-\widehat{M})$. Se $A$ é um p-grupo, então o resultado segue do Lema 2.3.1 (a demonstração dele também vale quando o corpo é $\mathbb{Q}$ ).

Se $A$ não é um $p$-grupo, então ele é produto direto de grupos de ordem potência de primos (distintos):

$$
A=G_{1} \ldots G_{n},
$$

onde cada $G_{i}$ é um $p_{i}$-grupo, e $p_{i} \neq p_{j}$, se $i \neq j$.

Assim, temos que $\varepsilon(A)=\varepsilon\left(G_{1}\right) \ldots \varepsilon\left(G_{n}\right)$. Para provar o que queremos, vamos provar que os idempotentes da forma $e_{1} \ldots e_{n}$, com $e_{i}$ um idempotente primitivo de $\mathbb{Q} G_{i}$, formam o conjunto dos idempotentes primitivos de $\mathbb{Q} G$.

Temos que a quantidade de idempotentes primitivos de $\mathbb{Q} G$ é dada pela quantidade de subgrupos cíclicos de $G$ que é, por sua vez, igual à quantidade de divisores de $|G|$, que é igual ao produto das quantidades de divisores de todos os $\left|G_{i}\right|$ 's, que coincide, por sua vez, com o produto da quantidade de idempotentes centrais primitivos de todos os $\mathbb{Q} G_{i}{ }^{\prime}$ s (pelo Teorema 1.4.9). Além disso todos, os idempotentes da forma $e_{1} \ldots e_{n}$, com $e_{i}$ um idempotente primitivo de $\mathbb{Q} G_{i}$, satisfazem as propriedades (i) e (ii) do Teorema 1.4.7, e a quantidade de tais idempotentes é a mesma que a quantidade de idempotentes centrais primitivos de $\mathbb{Q} G$. Vamos verificar a propriedade (iii) do Teorema 1.4.7:

Sejam $e_{1}^{i}, \ldots, e_{r_{i}}^{i}$ todos os idempotentes de $\mathbb{Q} G_{i}$, então $1_{G_{i}}=e_{1}^{i}+\ldots+e_{r_{i}}^{i}$. E temos $1=1_{G_{1}} \ldots 1_{G_{n}}=\left(e_{1}^{1}+\ldots+e_{r_{1}}^{1}\right) \ldots\left(e_{1}^{n}+\ldots+e_{r_{n}}^{n}\right)$, e este último é igual à soma de todos os idempotentes da forma $e_{1} \ldots e_{n}, \operatorname{com} e_{i}$ um idempotente primitivo de $\mathbb{Q} G_{i}$.

Portanto, temos um conjunto de idempotentes centrais 2 a 2 ortogonais que somam 1, e cuja quantidade é igual à de idempotentes centrais primitivos. Portanto, 
eles são os idempotentes centrais primitivos.

Usaremos agora os resultados anteriores para obter uma caracterização de todos os idempotentes centrais primitivos da álgebra de grupo racional de um grupo abeliano finito.

Corolário 3.2.7 Seja G um grupo abeliano finito. Os idempotentes centrais primitivos de $\mathbb{Q} G$ são precisamente os elementos da forma $\varepsilon(G, N)$, com $N$ um subgrupo de $G$ tal que $G / N$ é cíclico. Em particular, se e é um idempotente central primitivo, então supp (e) é um subgrupo de $G$, e e é uma combinação $\mathbb{Z}$-linear de idempotentes da forma $\widehat{H}$, onde $H$ é subgrupo de $G$.

Prova: Como já sabemos pelo lema anterior, se $A$ é cíclico, então $\varepsilon(A)$ é um idempotente primitivo de $\mathbb{Q} A$. Em particular, se $G / N$ é cíclico, temos que $\varepsilon(G / N)$ é idempotente primitivo de $\mathbb{Q}(G / N) \simeq(\mathbb{Q} G) \widehat{N}$ e, portanto, $\varepsilon(G, N)$ é um idempotente primitivo de $(\mathbb{Q} G) \widehat{N}$ e, portanto, de $\mathbb{Q} G$.

Seja agora e um idempotente primitivo de $\mathbb{Q} G$. Então $\left(G / G_{e}\right)_{\bar{e}}=\{1\}$ e, pelos lemas 3.2.4 e 3.2.5, $G / G_{e}$ é cíclico e $\varepsilon\left(G / G_{e}\right) \bar{e}=\bar{e}$, portanto, $\varepsilon\left(G / G_{e}\right)$ é idempotente primitivo de $\mathbb{Q}\left(G / G_{e}\right) \simeq \mathbb{Q} G\left(\widehat{G_{e}}\right)$ e, portanto, $\varepsilon\left(G, G_{e}\right)$ é idempotente central primitivo de $\mathbb{Q} G$, e também $\varepsilon\left(G, G_{e}\right) e=e$ e, portanto, $\varepsilon\left(G, G_{e}\right)=e$.

Provemos agora a segunda parte. Seja $e$ um idempotente primitivo de $\mathbb{Q} G$. Então temos que $e=\varepsilon\left(G, G_{e}\right)$. Se $M_{1}, \ldots, M_{n}$ são os subgrupos de $G$ que contém $G_{e}$ minimais, então temos que $e$ é combinação $\mathbb{Z}$-linear de $\widehat{G_{e}}$ e elementos do tipo $\widehat{G_{e}} \widehat{M_{i_{1}}} \ldots \widehat{M_{i_{k}}}=\widehat{M_{i_{1}}} \ldots \widehat{M_{i_{k}}}$.

Temos:

$$
\begin{gathered}
\widehat{M_{i_{1}}} \ldots \widehat{M_{i_{k}}}=\left(\frac{1}{\left|M_{i_{1}}\right|} \sum_{g \in M_{1}} g\right) \ldots\left(\frac{1}{\left|M_{i_{k}}\right|} \sum_{g \in M_{k}} g\right)= \\
=\frac{1}{\left|M_{i_{1}}\right| \ldots\left|M_{i_{k}}\right|}\left(\sum_{g \in M_{i_{1}}} g\right) \ldots\left(\sum_{g \in M_{i_{k}}} g\right)= \\
=\frac{1}{\left|M_{i_{1}}\right| \ldots\left|M_{i_{k}}\right|}\left(\left|G_{e}\right| \sum_{\bar{g} \in M_{i_{1}} / G_{e}} g \widehat{G_{e}}\right) \ldots\left(\left|G_{e}\right| \sum_{\bar{g} \in M_{i_{k}} / G_{e}} g \widehat{G_{e}}\right)=
\end{gathered}
$$




$$
=\frac{\left|G_{e}\right|^{k}}{\left|M_{i_{1}}\right| \ldots\left|M_{i_{k}}\right|}\left(\sum_{\bar{g} \in \frac{M_{i_{1}} \ldots M_{i_{k}}}{G_{e}}} g \widehat{G_{e}}\right)=\frac{1}{|M|} \sum_{g \in M} g=\widehat{M}
$$

onde $M=M_{i_{1}} \ldots M_{i_{k}}$.

Isso prova que e é combinação $\mathbb{Z}$-linear de elementos do tipo previsto.

Para provar que supp (e) é subgrupo de $G$, vamos novamente olhar para o quociente $\left(G / G_{e}\right)$, e vamos primeiro provar que $\operatorname{supp}(\bar{e})$ é subgrupo de $G / G_{e}$.

Como $G / G_{e}$ é cíclico, então ele é o produto direto de grupos cíclicos cujas ordens são potências de primos distintos

$$
G / G_{e}=P_{1} \times \ldots \times P_{r}
$$

e pela demonstração do Lema 3.2.6, temos que $\bar{e}=e_{1} \ldots e_{r}$, onde $e_{i}$ é um idempotente primitivo de $\mathbb{Q} P_{i}$. Portanto, $\operatorname{supp}(\bar{e})=\operatorname{supp}\left(e_{1}\right) \times \ldots \times \operatorname{supp}\left(e_{r}\right)$ e, portanto, podemos reduzir o problema ao caso em que $G / G_{e}$ é um $p$-grupo. Nesse caso, temos pelo Lema 2.3.1 do capítulo anterior que $\bar{e}=\widehat{G / G_{e}}$ ou $\bar{e}=\widehat{H}-\widehat{K}$, onde $H, K$ são subgrupos de $G / G_{e}$ e $H$ é subgrupo próprio de $K$. No primeiro caso, temos $\operatorname{supp}(\bar{e})=G / G_{e}$ e, no segundo caso, temos $\operatorname{supp}(\bar{e})=K$, e nosso resultado para $G / G_{e}$ está provado.

Portanto, novamente olhando para o isomorfismo $(\mathbb{Q} G) \widehat{G_{e}} \simeq \mathbb{Q}\left(G / G_{e}\right)$ (induzido pelo isomorfismo de grupos $g \widehat{G_{e}} \rightarrow \bar{g}$ ), temos que, se $e$ é um idempotente primitivo de $\mathbb{Q} G$ (e, como sabemos, de $(\mathbb{Q} G) \widehat{G_{e}}$ ), então o suporte de $\bar{e}$ é um subgrupo $\bar{M}$ de $G / G_{e}$ (portanto $\left.G_{e} \leq M\right)$, então o suporte de $e$ é

$$
\operatorname{supp}(e)=\bigcup_{g \in M} \operatorname{supp}\left(g \widehat{G_{e}}\right)=M \leq G,
$$

como queríamos provar.

Terminamos assim esta seção. 


\subsection{Grupos Nilpotentes Finitos}

O objetivo desta seção é generalizar o último corolário da seção anterior para o caso em que $G$ é um grupo nilpotente finito.

Vamos precisar introduzir uma notação:

Para um elemento $g \in G$, denotemos por $C_{g}$ o conjunto de todos os conjugados de $g$ por elementos de $G$, isto é: $C_{g}=\left\{h g h^{-1}: h \in G\right\}$, e é chamada de classe de conjugação de $g$.

Seja $x \in Z(\mathbb{Q} G)$ um elemento do centro de $\mathbb{Q} G$. Para todo $g \in g$ temos $g x g^{-1}=x$, isto implica que, para cada $h \in \operatorname{supp}(x)$, cada elemento de $C_{h}$ está no suporte de $x$ e tem o mesmo coeficiente de $h$.

Ou seja, acabamos de mostrar que os elementos $\widehat{C_{g}}$, com $g \in G$ formam uma $\mathbb{Q}$-base para o centro de $\mathbb{Q} G$.

Vamos provar um pequeno lema que será importante para provarmos o resultado principal:

Lema 3.3.1 Seja $G$ um grupo finito e $g \in G$. Se $g^{-1} C_{g} \cap Z(G) \neq\{1\}$, então $G$ contém um elemento central $z$, de ordem prima, tal que $\widehat{C_{g}}=\widehat{C_{g}} \widehat{\langle z\rangle}$.

Prova: Por hipótese, existe $h \in G$ tal que $g^{-1}\left(h^{-1} g h\right)=z \neq 1, z \in Z(G)$. Portanto, $g^{-1} h^{-1} g=z h^{-1}$.

Suponha que $|z|=p n$, onde $p$ é primo e $n$ é inteiro positivo. Então temos que

$$
z^{n} h^{-n}=\left(g^{-1} h^{-1} g\right)^{n}=g^{-1} h^{-n} g
$$

e portanto $1 \neq z^{n}=\left(g^{-1}\left(h^{n}\right)^{-1} g\right) h^{n} \in Z(G)$, e $\left|z^{n}\right|=p$. Portanto, podemos supor que $z$ tem ordem prima.

Novamente de $g^{-1}\left(h^{-1} g h\right)=z$, temos que

$$
h^{-1} g h=z g .
$$

Multiplicando por $h^{-(n-1)}$ à esquerda e por $h^{n-1}$ à direita da última equação, temos

$$
h^{-n} g h^{n}=h^{-(n-1)} z g h^{n-1}=z h^{-(n-1)} g h^{n-1}=
$$




$$
=z h^{-(n-2)} z g h^{n-2}=z^{2} h^{-(n-2)} g h^{n-2},
$$

e repetindo indutivamente o processo descrito acima mais $n-2$ vezes, temos que $h^{-n} g h^{n}=z^{n} g$.

Se $k \in G$, multiplicando a última equação por $k^{-1}$ à esquerda e por $k$ à direita, temos que

$$
k^{-1} h^{-n} g h^{n} k=\left(h^{n} k\right)^{-1} g\left(h^{n} k\right)=k^{-1} z^{n} g k=z^{n}\left(k^{-1} g k\right) .
$$

Daí, temos que $\langle z\rangle C_{g} \subseteq C_{g}$. A inclusão contrária é óbvia, pois $1 \in<z>$. Então temos $\langle z\rangle C_{g}=C_{g}$. Em particular, temos que as funções $f_{i}: C_{g} \rightarrow C_{g}$ definidas por $f_{i}(x)=z^{i} x$ são bijetoras. Daí, segue que $\widehat{C_{g}}=\widehat{C_{g}} \widehat{\langle z\rangle}$, pois $\widehat{C_{g}} \widehat{\langle z\rangle}=$ $\frac{1}{o(z)} \widehat{C_{g}}\left(z+\ldots+z^{o(z)}\right)=\frac{1}{o(z)} o(z) \widehat{C_{g}}=\widehat{C_{g}}$.

O centralizador de um subconjunto $S$ do grupo $G$ será denotado por $C_{G}(S)$, e é o cunjunto de todos os elementos de $G$ que comutam com todos os elementos de $S$. Prosseguiremos com o seguinte lema:

Lema 3.3.2 Seja $G$ um grupo finito e $N$ um subgrupo normal de $G$. Suponha que $e_{1}, \ldots, e_{n}$ é a lista de idempotentes centrais primitivos de $\mathbb{Q} N$, e seja $g \in G$. Então $g^{-1} e_{1} g, \ldots, g^{-1} e_{n} g$ também é a lista de idempotentes centrais primitivos de $\mathbb{Q} N$.

Prova: como $N$ é subgrupo normal de $G$, temos que a função $n \in N \mapsto g^{-1} n g \in$ $N$ é um automorfismo, que induz um automorfismo em $\mathbb{Q} N$, também dado pela conjugação pelo elemento $g \in G$. Assim, temos o resultado desejado.

A próxima proposição descreve os idempotentes centrais e da álgebra de grupo racional de um grupo nilpotente finito com $G_{e}=\{1\}$.

Proposição 3.3.3 Seja $G$ um grupo finito, e seja $e \in \mathbb{Q} G$. Se e é um idempotente central primitivo de $\mathbb{Q} G$, com $G_{e}=\{1\}$, então e é a soma de todos os $G$ conjugados de um idempotente central primitivo $e_{1} \in \mathbb{Q} G_{1}$, onde $G_{1}=C_{G}\left(Z_{2}(G)\right)$, e $\cap_{g \in G}\left(\left(G_{1}\right)_{e_{1}}\right)^{g}=1$. A reciproca vale se $G$ for nilpotente. 
Em particular, qualquer idempotente central primitivo e com $G_{e}$ trivial tem suporte contido em $C_{G}\left(Z_{2}(G)\right)$.

Prova: Suponha que $e \in \mathbb{Q} G$ é um idempotente central primitivo, com $G_{e}=\{1\}$. Seja $\mathcal{C}$ um conjunto de representantes de todas as classes de conjugação de $G$. Como já foi notado, $e$ pode ser escrito como $e=\sum_{g \in \mathcal{C}} \alpha_{g} \widehat{C_{g}}$, onde cada $\alpha_{g} \in \mathbb{Q}$.

Seja $g \notin C_{G}\left(Z_{2}(G)\right)$ um elemento de $G$. Seja $h^{-1} g h \in C_{g}$, com $h \in Z_{2}(G)$ tal que $h g \neq g h$. Temos que $g^{-1}\left(h^{-1} g h\right) \neq 1$.

Afirmação: $g^{-1}\left(h^{-1} g h\right) \in Z(G)$.

Para provar tal afirmação, olhemos para o quociente $G / Z(G)$ : neste quociente, temos que $\bar{h}$ e $\overline{h^{-1}}$ são centrais (pois $h \in Z_{2}(G)$ ). Então

$$
\overline{g^{-1}\left(h^{-1} g h\right)}=\overline{1}=Z(G) \Rightarrow g^{-1}\left(h^{-1} g h\right) \in Z(G)
$$

Assim, se $g \notin C_{G}\left(Z_{2}(G)\right)$, temos pelo Lema 3.3.1 que existe $w_{g} \in Z(G)$ de ordem prima tal que $\widehat{C_{g}}=\widehat{C_{g}} \widehat{\left\langle w_{g}\right\rangle}$. Além disso, como $Z_{2}(G)$ é normal em $G$, temos que $G_{1}=C_{G}\left(Z_{2}(G)\right)$ é normal e, portanto, os conjugados de elementos em $G_{1}$ estão em $G_{1}$. Então podemos escrever

$$
e=\sum_{g \in C_{G}\left(Z_{2}(G)\right) \cap \mathcal{C}} \alpha_{g} \widehat{C_{g}}+\sum_{g \notin C_{G}\left(Z_{2}(G)\right), g \in \mathcal{C}} \alpha_{g} \widehat{C_{g}} \widehat{\left\langle w_{g}\right\rangle}
$$

Como $G_{e}$ é trivial, pelo Lema 3.2.4, temos que $e \varepsilon(G)=e$. Mas como cada $\left\langle w_{g}\right\rangle$ é normal minimal, temos

$$
\varepsilon(G) \widehat{\left\langle w_{g}\right\rangle}=\left(\prod_{M \in \mathcal{M} \backslash\left\{\left\langle w_{g}\right\rangle\right\}}(1-\widehat{M})\right)\left(1-\widehat{\left\langle w_{g}\right\rangle}\right) \widehat{\left\langle w_{g}\right\rangle}=0 .
$$

Portanto, podemos escrever

$$
e=\sum_{g \in C_{G}\left(Z_{2}(G)\right) \cap \mathcal{C}} \alpha_{g} \widehat{C_{g}} \cdot \varepsilon(G)
$$

Analogamente, podemos escrever

$$
\varepsilon(G)=\sum_{g \in C_{G}\left(Z_{2}(G)\right) \cap \mathcal{C}} \beta_{g} \widehat{C_{g}}+\sum_{g \notin C_{G}\left(Z_{2}(G)\right), g \in \mathcal{C}} \beta_{g} \widehat{C_{g}} \widehat{\left\langle w_{g}\right\rangle}
$$


e como $\varepsilon(G) \widehat{\left\langle w_{g}\right\rangle}=0$, temos:

$$
0=\sum_{g \in C_{G}\left(Z_{2}(G)\right) \cap \mathcal{C}} \beta_{g} \widehat{C_{g}} \widehat{\left\langle w_{g}\right\rangle}+\sum_{g \notin C_{G}\left(Z_{2}(G)\right), g \in \mathcal{C}} \beta_{g} \widehat{C_{g}} \widehat{\left\langle w_{g}\right\rangle},
$$

e como $C_{G}\left(Z_{2}(G)\right)$ é normal em $G$ e $\left\langle w_{g}\right\rangle \subset C_{G}\left(Z_{2}(G)\right)=G_{1}$, temos

$$
\begin{gathered}
\operatorname{supp}\left(\sum_{g \in C_{G}\left(Z_{2}(G)\right) \cap \mathcal{C}} \eta_{g} \widehat{C_{g}} \widehat{\left\langle w_{g}\right\rangle}\right) \subset C_{G}\left(Z_{2}(G)\right), \\
\operatorname{supp}\left(\sum_{g \notin C_{G}\left(Z_{2}(G)\right), g \in \mathcal{C}} \eta_{g} \widehat{C_{g}} \widehat{\left\langle w_{g}\right\rangle}\right) \cap C_{G}\left(Z_{2}(G)\right)=\emptyset,
\end{gathered}
$$

para quaisquer $\eta_{g} \in \mathbb{Q}$.

Em particular, temos, pela equação (3.3) que $\sum_{g \notin C_{G}\left(Z_{2}(G)\right), g \in \mathcal{C}} \beta_{g} \widehat{C_{g}} \widehat{\left\langle w_{g}\right\rangle}=0 \mathrm{e}$, portanto, $\operatorname{supp}(\varepsilon(G)) \subset C_{G}\left(Z_{2}(G)\right)$.

Aplicando o parágrafo anterior na equação (3.2), temos que supp $(e) \subset C_{G}\left(Z_{2}(G)\right)=$ $G_{1}$. Ou seja, $e \in \mathbb{Q} G_{1}$.

Não temos certeza se e é idempotente central primitivo em $\mathbb{Q} G_{1}$, mas com certeza é idempotente central. Portanto, podemos escrever

$$
e=e_{1}+\ldots+e_{n}
$$

onde cada $e_{i}$ é um idempotente central primitivo de $\mathbb{Q} G_{1}$.

Seja $g \in G$. Temos que

$$
e=e^{g}=e_{1}^{g}+\ldots+e_{n}^{g}
$$

Seja $\widetilde{e}=e_{1}^{g_{1}}+\ldots+e_{1}^{g_{k}}$ a soma de todas as $G$-classes de conjugação de $e_{1}$. Temos que $\widetilde{e}$ é central em $\mathbb{Q} G$. E pelo Lema 3.3.2, podemos concluir que $\widetilde{e}$ é idempotente e não nulo.

Assim sendo, $\widetilde{e} e=e$ ou 0. Usando a equação (3.4) temos:

$\widetilde{e} e=e_{1}^{g_{1}} e+\ldots+e_{1}^{g_{k}} e=e_{1}^{g_{1}}\left(e_{1}^{g_{1}}+\ldots+e_{n}^{g_{1}}\right)+\ldots+e_{1}^{g_{k}}\left(e_{1}^{g_{k}}+\ldots+e_{n}^{g_{k}}\right)=e_{1}^{g_{1}}+\ldots+e_{1}^{g_{k}}=\widetilde{e} \neq 0$, 
desta equação, temos que $e=\widetilde{e}$ (isso prova uma parte da proposição, e também prova que $n=k)$.

Como $\left(\left(G_{1}\right)_{e_{1}}\right)^{g_{i}}=\left(G_{1}\right)_{e_{1}^{g_{i}}}$, então segue que $\cap_{i=1}^{n}\left(\left(G_{1}\right)_{e_{1}}\right)^{g_{i}}=\cap_{i=1}^{n}\left(G_{1}\right)_{e_{1}^{g_{i}}} \subseteq G_{e}=$ $\{1\}$ e segue que $\cap_{i=1}^{n}\left(\left(G_{1}\right)_{e_{1}}\right)^{g_{i}}=\{1\}$. Assim, provamos um dos lados da proposição.

Por outro lado, suponha que $e_{1}$ é um idempotente central primitivo de $\mathbb{Q} G_{1}$, com $G_{1}=C_{G}\left(Z_{2}(G)\right)$ e suponha que $\cap_{i=1}^{n}\left(\left(G_{1}\right)_{e_{1}}\right)^{g_{i}}=\{1\}$.

Seja $e=e_{1}^{g_{1}}+\ldots+e_{1}^{g_{n}}$ a soma de todas as $G$-classes de conjugação de $e_{1}$.

Temos que e é central em $\mathbb{Q} G$ e, além disso, concluímos pelo Lema 3.3.2 que e é idempotente. Portanto, existem $f_{1}, \ldots, f_{k}$ idempotentes centrais primitivos em $\mathbb{Q} G$ tais que $e=f_{1}+\ldots+f_{k}$.

Seja $N$ um subgrupo central não trivial de $G$. Temos que $\widehat{N}$ é um idempotente central em $\mathbb{Q} G_{1}$ e, portanto, $\widehat{N} e_{1}=0$ ou $e_{1}$, sendo que se for igual a $e_{1}$, implica $N \subseteq\left(G_{1}\right)_{e_{1}}$ (pelo Lema 3.2.1) e então

$$
N \subseteq \bigcap_{g \in G}\left(\left(G_{1}\right)_{e_{1}}\right)^{g}=\{1\}
$$

o que não é possível.

Sendo assim, $\widehat{N} e_{1}=0$. Como $\left(G_{1}\right)_{e_{1}}$ é normal em $G$ e $G$ é grupo nilpotente, temos que existe subgrupo $N \neq\{1\}$ de $G$, contido em $Z(G) \cap\left(G_{1}\right)_{e_{1}}$, se $\left(G_{1}\right)_{e_{1}} \neq\{1\}$.

Mas como $\widehat{N} e_{1}=0$, então $\left(G_{1}\right)_{e_{1}}=\{1\}$ e, pelo Lema 3.2.4, temos que $\varepsilon\left(G_{1}\right) e_{1}=$ $\varepsilon(G) e_{1}=e_{1}$ (obs.: $\varepsilon(G)=\varepsilon\left(G_{1}\right)$, pois $G$ é nilpotente e também $\left.Z(G) \subseteq G_{1}\right)$.

Consequentemente, temos $\varepsilon(G) e=e$ e, portanto, $\varepsilon(G) f_{i}=f_{i}$, para todo $i$. Pelo Lema 3.2.4, temos que $G_{f_{i}}$ é trivial e, portanto, pela primeira parte da prova, temos $f_{i} \in \mathbb{Q} G_{1}$.

Como $e_{1}$ aparece na representação de $e$ como soma de idempotentes centrais primitivos de $\mathbb{Q} G_{1}$, então $e_{1}$ também aparece na mesma representação de algum $f_{i}$. Pela primeira parte da prova, temos que $f_{i}=e_{1}^{g_{1}}+\ldots+e_{1}^{g_{n}}=e$, portanto, $e=f_{i}$ é idempotente central primitivo de $\mathbb{Q} G$, como queríamos.

Agora vamos ao resultado principal deste capítulo, onde descreveremos os idempotentes primitivos centrais de quaisquer álgebras racionais de grupos nilpotentes 
finitos, dando uma descrição que depende apenas da estrutura do reticulado de subgrupos do grupo nilpotente em questão.

Teorema 3.3.4 Seja $G$ um grupo nilpotente finito. Então os idempotentes centrais primitivos são precisamente todos os elementos da forma

$$
\left(\varepsilon\left(G_{m}, H_{m}\right)\right)^{g_{1}}+\ldots+\left(\varepsilon\left(G_{m}, H_{m}\right)\right)^{g_{n}}
$$

isto é, a soma de todos os $G$-conjugados de $\varepsilon\left(G_{m}, H_{m}\right)$, onde $H_{m}, G_{m}$ são subgrupos de $G$ que satisfazem às seguintes propriedades:

(1) $H_{0} \subseteq H_{1} \subseteq \ldots \subseteq H_{m} \subseteq G_{m} \subseteq \ldots \subseteq G_{1} \subseteq G_{0}=G$;

(2) para $0 \leq i \leq m, H_{i}$ é um subgrupo normal de $G_{i}$ e $Z\left(G_{i} / H_{i}\right)$ é cíclico;

(3) para $0 \leq i<m, G_{i} / H_{i}$ não é abeliano, $e G_{m} / H_{m}$ é abeliano;

(4) para $0 \leq i<m, G_{i+1} / H_{i}=C_{G_{i} / H_{i}}\left(Z_{2}\left(G_{i} / H_{i}\right)\right)$;

(5) para $1 \leq i \leq m, \cap_{x \in G_{i-1} / H_{i-1}} H_{i}^{x}=H_{i-1}$.

Prova: Em primeiro lugar, vamos ver que um elemento do tipo acima é mesmo um idempotente central primitivo.

Seja $e=\left(\varepsilon\left(G_{m}, H_{m}\right)\right)^{g_{1}}+\ldots+\left(\varepsilon\left(G_{m}, H_{m}\right)\right)^{g_{n}}$ satisfazendo às propriedades acima. Por causa do Corolário 3.2.7, temos que as propriedades (2) e (3) implicam que $\overline{f_{m}}=\varepsilon\left(G_{m} / H_{m}\right)$ é um idempotente (central) primitivo de $\mathbb{Q}\left(G_{m} / H_{m}\right) \simeq\left(\mathbb{Q} G_{m}\right) \widehat{H_{m}}$. Daí, temos que $\varepsilon\left(G_{m}, H_{m}\right)=f_{m}$ é um idempotente central primitivo de $\mathbb{Q} G_{m}$.

Como $\varepsilon\left(G_{m} / H_{m}\right) \overline{f_{m}}=\overline{f_{m}}$, então $\left(G_{m} / H_{m}\right)_{\overline{f_{m}}}=\{\overline{1}\}$, pelo Lema 3.2.4. Lembrando que $\mathbb{Q}\left(G_{m} / H_{m}\right) \simeq\left(\mathbb{Q} G_{m}\right) \widehat{H_{m}}$ pelo isomorfismo induzido pelo isomorfismo de grupos $g \in G \widehat{H_{m}} \rightarrow \bar{g} \in G / H_{m}$, temos:

$$
\begin{gathered}
g \in\left(G_{m}\right)_{f_{m}} \Leftrightarrow g f_{m}=\left(g \widehat{H_{m}}\right)\left(f_{m} \widehat{H_{m}}\right)=f_{m} \widehat{H_{m}}=f_{m} \Leftrightarrow \\
\Leftrightarrow \bar{g} \in\left(G_{m} / H_{m}\right)_{\overline{f_{m}}}=\{\overline{1}\} \Leftrightarrow g \in H_{m} .
\end{gathered}
$$

Assim, temos que $\left(G_{m}\right)_{f_{m}}=H_{m}$. 
A propriedade (4) implica que, em particular, $G_{i+1} / H_{i}$ é subgrupo normal de $G_{i} / H_{i}$ e, usando o teorema da correspondência, temos que $G_{m}$ é normal em $G_{m-1}$.

Portanto temos, pelo Lema 3.3.2, que $f_{m-1}$ a soma de todos os $G_{m-1}$-conjugados de $f_{m}$ é idempotente, além disso, $f_{m-1}$ é claramente central em $\mathbb{Q} G_{m-1}$. A propriedade (5) nos dá

$$
\begin{gathered}
\bigcap_{g \in G_{m-1}}\left(\left(G_{m}\right)_{f_{m}}\right)^{g}=\bigcap_{g \in G_{m-1}} H_{m}^{g}=H_{m-1} \\
\Rightarrow \bigcap_{g \in G_{m-1} / H_{m-1}}\left(\left(G_{m} / H_{m-1}\right)_{\overline{f_{m}}}\right)^{g}=\bigcap_{g \in G_{m-1} / H_{m-1}}\left(H_{m} / H_{m-1}\right)^{g}=\{1\} .
\end{gathered}
$$

Assim, as propriedades (2), (4) e a fórmula acima, junto com a proposição anterior implicam que $f_{m-1}$ é um idempotente central primitivo de $\left(\mathbb{Q} G_{m-1}\right) \widehat{H_{m-1}} \simeq$ $\mathbb{Q}\left(G_{m-1} / H_{m-1}\right)$ (olhando primeiro para o quociente). Portanto, $f_{m-1}$ é idempotente primitivo central de $\mathbb{Q} G_{m-1}$.

Repetindo o argumento acima para $f_{m-2}$ a soma de todos os $G_{m-2}$-conjugados de $f_{m-1}$, temos que $f_{m-2}$ é idempotente central primitivo de $\mathbb{Q} G_{m-2}$. A única parte da prova que não é análogo ao que fizemos, é para provar que $\left(G_{m-1}\right)_{f_{m-1}}=H_{m-1}$. Para isso, vamos escrever $f_{m-1}=f_{m}^{g_{1}}+\ldots+f_{m}^{g_{k}}$. Assim,

$$
h \in\left(G_{m-1}\right)_{f_{m-1}} \Leftrightarrow f_{m-1} h=f_{m-1}
$$

como o suporte de $f_{m-1}$ está contido em $G_{m}$, então temos $h \in G_{m}$. Portanto, pelo Lema 3.3.2 e o Teorema de Wedderburn-Artin, temos que vale (3.5) se, e somente se $f_{m}^{g_{i}} h=f_{m}^{g_{i}}$, para todo $i$, que equivale a dizer que $f_{m} h^{g_{i}^{-1}}=f_{m}$ e, portanto, $h^{g_{i}^{-1}} \in H_{m}$ e, portanto $h \in H_{m}^{g_{i}}$. Como a escolha de $f_{m}$ perite que $g_{i}$ seja qualquer elemento de $G_{m-1}$ temos, pela propriedade (5) que $h \in H_{m-1}$. Portanto, provamos assim que $\left(G_{m-1}\right)_{f_{m-1}}=H_{m-1}$.

Repetindo esse processo indutivamente (para $f_{m-3}, f_{m-4}, \ldots f_{0}$ ), chegaremos que $f_{0}$ será idempotente central primitivo de $\mathbb{Q} G_{0}=\mathbb{Q} G$.

Basta provar agora que $f_{0}=e$ : para isso, vamos provar que $f_{m-2}$ é a soma de todos os $G_{m-2}$-conjugados de $f_{m}=\varepsilon\left(G_{m}, H_{m}\right)$, e o restante sai trivialmente por indução (seguindo os mesmos passos).

Vamos escrever $f_{m-1}=f_{m}^{h_{1}}+\ldots+f_{m}^{h_{k}}$ (soma de todos os $G_{m-1}$-conjugados de 
$\left.f_{m}\right), f_{m-2}=f_{m-1}^{s_{1}}+\ldots+f_{m-1}^{s_{l}}$ (soma de todos os $G_{m-2}$-conjugados de $\left.f_{m-1}\right)$. Então, temos que

$$
f_{m-2}=\sum_{1 \leq i \leq k ; 1 \leq j \leq l} f_{m}^{h_{i} s_{j}}
$$

Seja $x \in G_{m-2}$. Existe $j$ tal que $f_{m-1}^{s_{j}}=f_{m-1}^{h_{1}^{-1} x}$ e, portanto, $f_{m}^{x}$ é um termo da equação (3.6).

Agora basta provar que cada $G_{m-2}$-conjugado de $f_{m}$ aparece uma única vez na soma (3.6). Para isso, vamos provar que são todos ortogonais:

Usando o mesmo argumento da prova do Lema 3.3.2, concluímos que $f_{m}^{h_{a} s_{b}} f_{m}^{h_{c} s_{b}}=$ 0 (podemos fazer isso, pois a propriedade (4) implica que $G_{i+1}$ é subgrupo normal de $\left.G_{i}\right)$, se $a \neq c$ e, além disso, pelo Lema 3.3.2, temos que $\left(\sum_{1 \leq i \leq k} f_{m}^{h_{i} s_{b}}\right)\left(\sum_{1 \leq i \leq k} f_{m}^{h_{i} s_{d}}\right)=$ 0 , se $b \neq d$. Daí, temos:

$$
f_{m}^{h_{\alpha} s_{b}} f_{m}^{h_{\beta} s_{d}}=\left(f_{m}^{h_{\alpha} s_{b}}\left(\sum_{1 \leq i \leq k} f_{m}^{h_{i} s_{b}}\right)\right)\left(\left(\sum_{1 \leq i \leq k} f_{m}^{h_{i} s_{d}}\right) f_{m}^{h_{\beta} s_{d}}\right)=0 .
$$

Continuando indutivamente com este argumento, concluímos que $f_{m-i}$ é a soma de todos os $G_{m-i^{-}}$conjugados de $f_{m}$ e, portanto, concluímos que $f_{0}=e$. (Obs.: além disso, também temos que os $G$-conjugados de $f_{m}$ são ortogonais entre si).

Agora vamos provar que todo idempotente central primitivo é dessa forma.

Seja $e \in \mathbb{Q} G$ um idempotente central primitivo de $\mathbb{Q} G$. Tomemos $H_{0}=G_{e}$ subgrupo normal de $G=G_{0}$ e temos que e é idempotente central primitivo de $(\mathbb{Q} G) \widehat{H_{0}} \simeq \mathbb{Q}\left(G_{0} / H_{0}\right)$.

Temos que $\left(G_{0} / H_{0}\right)_{\bar{e}}=\{1\}$. Como já sabemos, isto implica $\varepsilon\left(G_{0} / H_{0}\right) \neq 0$, que por sua vez, implica $Z\left(G_{0} / H_{0}\right)$ cíclico. Portanto, se $G_{0} / H_{0}$ for abeliano, então será cíclico. Pelo Corolário 3.2.7 teremos $\varepsilon\left(G_{0}, H_{0}\right)$ é idempotente central primitivo de $\mathbb{Q} G_{0}$ e, como $e=e \varepsilon\left(G_{0}, H_{0}\right)$, então $e=\varepsilon\left(G_{0}, H_{0}\right)$, que é do tipo desejado (isto é, é do tipo que satisfaz às propriedades do enunciado do teorema).

Se $G_{0} / H_{0}$ não for abeliano, ainda teremos que o seu centro é cíclico. Segue da proposição anterior que $\bar{e} \in \mathbb{Q}\left(G_{0} / H_{0}\right)$ é a soma de todos os $\left(G_{0} / H_{0}\right)$-conjugados de um 
idempotente central primitivo $e_{1}$ de $\mathbb{Q}\left(G_{1} / H_{0}\right) \simeq\left(\mathbb{Q} G_{1}\right) \widehat{H_{0}}$ (através desse isomorfismo, podemos dizer que é a soma dos $G$-conjugados de $\left.e_{1}\right)$, onde $G_{1}$ é um subgrupo de $G$ tal que $G_{1} / H_{0}=C_{G_{0} / H_{0}}\left(Z_{2}\left(G_{0} / H_{0}\right)\right) \neq G_{0} / H_{0}$, e $\cap_{x \in G_{0} / H_{0}}\left(H_{1} / H_{0}\right)^{x}=1$, com $H_{1}$ um subgrupo de $G$ contendo $H_{0}$ tal que $H_{1} / H_{0}=\left(G_{1} / H_{0}\right)_{e_{1}} \triangleleft G_{1} / H_{0}$ (isso tudo nós temos pela proposição anterior!). Isto implica $H_{1} \triangleleft G_{1}$ e, como $\left(G_{1} / H_{1}\right)_{e_{1}}=\{1\}$, temos que $Z\left(G_{1} / H_{1}\right)$ é cíclico. Portanto, se $\left(G_{1} / H_{1}\right)$ é abeliano, então é cíclico (pois será igual ao seu centro, que é cíclico). Pelo Corolário 3.2.7, temos que $e_{1}=\varepsilon\left(G_{1}, H_{1}\right)$ e segue o resultado, pois verificamos as propriedades de (1) a (5). Antes de continuarmos indutivamente, observemos o seguinte:

Obs.: temos que a classe de nilpotência de $\left(G_{1} / H_{0}\right)$ é sempre estritamente menor que a de $\left(G_{0} / H_{0}\right)$, pois $\left(G_{1} / H_{0}\right)$ centraliza o segundo centro de $\left(G_{0} / H_{0}\right)$. Além disso, como $\left(G_{1} / H_{1}\right)$ é isomorfo a um quociente de $\left(G_{1} / H_{0}\right)$, então se $\left(G_{1} / H_{0}\right)$ for abeliano, $\left(G_{1} / H_{1}\right)$ também será.

Agora continuemos com o próximo passo:

Se $\left(G_{1} / H_{1}\right)$ não for abeliano, continuemos indutivamente da seguinte maneira: Já começaremos com $e_{i} \in \mathbb{Q}\left(G_{i} / H_{i}\right) \simeq\left(\mathbb{Q} G_{i}\right) \widehat{H_{i}}$ um idempotente central primitivo, e repetindo o argumento acima, obteremos que $e_{i}$ é a soma de conjugados por $G_{i}$ de um idempotente central primitivo $e_{i+1}$ de $\left(G_{i+1} / H_{i}\right)$, onde $G_{i+1}$ é obtido de forma análoga à que obtivemos $G_{1}$ acima, e também obteremos analogamente $H_{i+1}$ e, novamente, se $G_{i+1} / H_{i+1}$ for abeliano, acaba (com as propriedades (1) a (5) verificadas neste passo e nos anteriores) e, se não for, repete o processo para $e_{i+1} \in\left(\mathbb{Q} G_{i+1}\right) \widehat{H_{i+1}}$.

Este processo acabará pois, temos que a classe de nilpotência de $G_{i+1} / H_{i}$ é estritamente menor que a de $G_{i} / H_{i-1}$, pois

$$
G_{i+1} / H_{i}=C_{G_{i} / H_{i}}\left(Z_{2}\left(G_{i} / H_{i}\right)\right)
$$

onde $G_{i} / H_{i}$ é isomorfo a um quociente de $G_{i} / H_{i-i}$. E como já observamos de forma análoga acima, $G_{i+1} / H_{i}$ abeliano implica $G_{i+1} / H_{i+1}$ abeliano.

Quando o processo acabar, escreveremos e como uma soma do mesmo tipo que $f_{0}$ (da primeira parte da prova), ou seja, e é a soma de todos os $G$-conjugados de 
$\varepsilon\left(G_{m}, H_{m}\right)$.

E o teorema está provado.

Segue da demonstração deste teorema que todos os $G$-conjugados de $\varepsilon\left(G_{m}, H_{m}\right)$ são dois a dois ortogonais.

E temos pela demonstração do Corolário 3.2.7 que os idempotentes $\varepsilon\left(G_{m}, H_{m}\right)$ são uma combinação $\mathbb{Z}$-linear de elementos do tipo $\widehat{H}$, com $H$ subgrupo de $G$ (pois $G_{m} / H_{m}$ é cíclico).

Então, temos de forma imediata o seguinte corolário, que encerra esta seção:

Corolário 3.3.5 Seja $G$ um grupo nilpotente finito. Se e é um idempotente central primitivo de $\mathbb{Q} G$, então e é combinação $\mathbb{Z}$-linear de idempotentes do tipo $\widehat{H}$, onde $H$ é um subgrupo de $G$.

\subsection{Calculando um Exemplo concreto}

Como vimos no capítulo introdutório, os grupos multiplicativos $G=U T\left(n, F_{q}\right)$ (das matrizes unitriangulares $n \times n$ com entradas no corpo $F_{q}$ ) são todas nilpotentes de classe $(n-1)$, e que $Z_{i}(G)$ é o subgrupo composto das matrizes que têm entradas nulas nas $(n-i-1)$ diagonais logo acima da diagonal principal.

Nosso objetivo ao longo desta seção será utilizar o teorema principal da seção anterior para calcular os idempotentes centrais primitivos de $\mathbb{Q} G^{p}:=\mathbb{Q}\left(U T\left(3, F_{p}\right)\right)$, para todo $p$ primo, utilizando para isso as relações que nós temos entre os subgrupos de $U T\left(3, F_{p}\right)$.

Sejam $\left(G_{i}\right),\left(H_{i}\right)$ sequências de subgrupos de $G^{p}$ que satisfazem às condições do Teorema 3.3.4. Como $\left|G^{p}\right|=p^{3}$, temos que $\left|G_{m}\right|=1, p, p^{2}$ ou $p^{3}$.

Primeiro caso: se $\left|G_{m}\right|<p^{3}$, então como $\frac{G_{m-1}}{H_{m-1}}$ não é abeliano (condição (3)), então $G_{m-1}=G^{p}$ e $H_{m-1}=\{1\}$ (pois todo grupo de ordem $p$ ou $p^{2}$ é abeliano). 
Além disso, como o grau de nilpotência de $G^{p}$ é 2 , então o de $G_{m-1} / H_{m-1}$ é menor ou igual a 2. Daí, temos:

$$
C_{\frac{G_{m-1}}{H_{m-1}}}\left(Z_{2}\left(G_{m-1} / H_{m-1}\right)\right)=C_{\frac{G^{p}}{\{1\}}}\left(\frac{G^{p}}{\{1\}}\right)=Z(G) /\{1\}
$$

de onde, pela propriedade (4), tiramos que $G_{m}=Z(G)$ um grupo de ordem $p$. Assim temos, a princípio, duas possibilidades para $H_{m}: H_{m}=\{1\}$ ou $H_{m}=G_{m}=Z(G)$. O segundo caso nos dá:

$$
\bigcap_{x \in \frac{G_{m-1}}{H_{m-1}}=\frac{G^{p}}{G^{p}}} H_{m}^{x}=H_{m}=Z(G) \neq H_{m-1}=\{1\},
$$

contradizendo a propriedade (5). Mas no caso $H_{m}=\{1\}$ temos:

$$
\bigcap_{x \in \frac{G_{m-1}}{H_{m-1}=\frac{G p}{\{1\}}}} H_{m}^{x}=\{1\}=H_{m-1}
$$

Além disso, $Z\left(G_{m-1} / H_{m-1}\right)=Z\left(G^{p} /\{1\}\right) \simeq Z\left(G^{p}\right)$ é cíclico (satisfaz (2)). Portanto temos que a sequência $H_{0}=\{1\} \subset H_{1}=\{1\} \subset G_{1}=Z\left(G^{p}\right) \subset G_{0}=G^{p}$ satisfaz as hipóteses do Teorema 3.3.4.

Portanto, $\varepsilon\left(Z\left(G^{p}\right),\{1\}\right)=1-\widehat{Z\left(G^{p}\right)}$ é idempotente central primitivo de $\mathbb{Q} G^{p}$.

Segundo caso: se $\left|G_{m}\right|=p^{3}$. Vamos primeiro fazer um cálculo:

Seja $A=\left(\begin{array}{ccc}1 & a_{12} & a_{13} \\ 0 & 1 & a_{23} \\ 0 & 0 & 1\end{array}\right) \in G^{p}$. Temos facilmente, por indução, que

$$
A^{n}=\left(\begin{array}{ccc}
1 & n a_{12} & n a_{13}+\frac{p(p-1)}{2} a_{12} a_{23} \\
0 & 1 & n a_{23} \\
0 & 0 & 1
\end{array}\right)
$$

Portanto temos 2 subcasos a considerar: 
$p \neq 2$ : nesse caso, temos que $A^{p}=I$. Sendo assim, se $a_{12} \neq 0$ ou $a_{23} \neq 0$, então $\langle A\rangle$ é grupo cíclico de ordem $p$ que não intersecta o centro, portanto, não pode ser normal. O outro subgrupo de ordem $p$ é o próprio $Z(G)$, que é gerado por $g=\left(\begin{array}{ccc}1 & 0 & 1 \\ 0 & 1 & 0 \\ 0 & 0 & 1\end{array}\right) \in G_{p}$.

Também temos subgrupos de ordem $p^{2}$ (são todos normais, pois o quociente tem ordem prima): os gerados por $A$ e $g$, onde $g$ está definido logo acima e $A$ é tal que $a_{12}$ ou $a_{23}$ é não nulo (podemos supor que $a_{13}=0$ ). Temos $p+1$ subgrupos de ordem $p^{2}$ no total (os casos em que $a_{12}=1$ e $a_{23}$ percorre $F_{p}$ juntos com o caso em que $a_{12}=0$ e $a_{23}=1$ são todos os casos possíveis).

Assim, temos uma descrição completa de todos os subgrupos de $G^{p}$ (quando $p$ é ímpar).

Lembramos que estamos no caso $G_{m}=G^{p}$. Pela propriedade (2), temos que $H_{m}$ é normal em $G_{m}$. Assim, temos, a princípio, três possibilidades para $H_{m}: H_{m}=$ $Z\left(G^{p}\right),\{1\}$ ou tem ordem $p^{2}$. Os dois primeiros casos não satisfazem às propriedades (2) e (3) pois $G_{m} / H_{m}$ não será cíclico. Portanto, o único caso que ainda pode acontecer é $H_{m}$ ter ordem $p^{2}$. Nesse caso, a sequência $H_{0} \subset G_{0}($ com m $=0)$ satisfaz às propriedades do Teorema 3.3.4.

Portanto, temos que $\varepsilon\left(G_{m}, H_{m}\right)=\varepsilon\left(G^{p}, H_{m}\right)=\widehat{H_{m}}-\widehat{G^{p}}$ são idempotentes centrais primitivos de $\mathbb{Q} G^{p}$.

Assim, terminamos de calcular todos os $(p+2)$ idempotentes centrais primitivos de $\mathbb{Q} G^{p}$ no caso de $p$ ser primo ímpar:

$$
\begin{gathered}
e_{1}=\left(1-\widehat{Z\left(G^{p}\right)}\right) \\
e_{i}=\widehat{H_{i}}-\widehat{G^{p}}
\end{gathered}
$$

onde $H_{i}$ é subgrupo de ordem $p^{2}$ de $G^{p}$ (há $(p+1)$ tais subgrupos).

$p=2$ : pelos cálculos feitos acima, temos um subgrupo cíclico de ordem 4 gerado 
por

$$
h=\left(\begin{array}{lll}
1 & 1 & 0 \\
0 & 1 & 1 \\
0 & 0 & 1
\end{array}\right)
$$

Também temos 5 subgrupos de ordem 2, sendo um deles $Z\left(G^{2}\right)$ e os outros quatro são os gerados por:

$$
\begin{aligned}
& \left(\begin{array}{ccc}
1 & 1 & a_{13} \\
0 & 1 & 0 \\
0 & 0 & 1
\end{array}\right), a_{13} \in F_{2} \\
& \left(\begin{array}{ccc}
1 & 0 & a_{13} \\
0 & 1 & 1 \\
0 & 0 & 1
\end{array}\right), a_{13} \in F_{2}
\end{aligned}
$$

Também temos mais 2 subgrupos (não-cíclicos) de ordem 4, gerados por um dos elementos acima e por $g=\left(\begin{array}{lll}1 & 0 & 1 \\ 0 & 1 & 0 \\ 0 & 0 & 1\end{array}\right)$ (nesse caso, pode supor $a_{13}=0$, por isso que são apenas 2 subgrupos desse tipo).

Lembremos que estamos no caso $G_{m}=G^{2}$. Pelas propriedades (2) e (3) do Teorema 3.3.4, temos que $\left(G_{m} / H_{m}\right)$ é cíclico e, portanto, os únicos candidatos a $H_{m}$ possíveis são quando $\left|H_{m}\right|=4$ (3 casos), ou $H_{m}=G_{m}$ (1 caso).

Em todos os casos listados acima, a sequência $H_{0} \subset G_{0}$ (aqui $m=0$ ) satisfaz as hipóteses do Teorema 3.3.4.

Portanto, encontramos assim os 5 idempotentes centrais primitivos de $\mathbb{Q} G^{2}$ :

$$
\begin{gathered}
\widehat{G} ; \\
\widehat{H_{i}}-\widehat{G}, \\
1-\widehat{Z\left(G^{2}\right)}
\end{gathered}
$$

onde $\left|H_{i}\right|=4$ (há 3 subgrupos com 4 elementos, conforme vimos acima). 
Assim, pudemos obter a partir das relações entre os subgrupos de $G^{p}=U T\left(3, F_{p}\right)$, usando o Teorema 3.3.4, todos os idempotentes centrais primitivos de $\mathbb{Q}\left(U T\left(3, F_{p}\right)\right)$, para todo $p$ primo, concluindo assim este capítulo. 
64 IDEMPOTENTES CENTRAIS PRIMITIVOS EM ÁLGEBRAS DE GRUPO RACIONAIS DE GRUPOS NILPOTENTES FINITOS 


\section{Capítulo 4}

\section{Conclusões}

Chegamos, assim, ao fim do objetivo principal do nosso trabalho.

\subsection{Considerações Finais}

No capítulo introdutório, pudemos ver alguns dos resultados e conceitos básicos da teoria de anéis de grupos e algumas aplicações da teoria no estudo de códigos cíclicos. Também vimos alguns conceitos básicos necessários acerca de caracteres em grupos abelianos finitos e acerca de grupos nilpotentes.

No capítulo seguinte, foi estudado um artigo em que, dentre outras coisas, estendeu resultados antes obtidos por Arora e Pruthi [6], por calcular idempotentes geradores de códigos abelianos minimais de certas álgebras de grupo.

No capítulo seguinte, estudamos uma descrição dos idempotentes centrais primitivos de álgebras racionais de grupos nilpotentes finitos, sem necessitar de usar a tabela de caracteres do grupo. Além disso, neste mesmo capítulo, também estudamos uma descrição dos idempotentes primitivos de álgebras racionais de grupos abelianos, diferente da que é feita por [1, Teorema vii.1.4], e concluindo logo em seguida que o suporte desses idempotentes são subgrupos do grupo abeliano em questão, além de esses idempotentes poderem ser escritos como uma soma de termos de um tipo particular.

Neste capítulo também listamos alguns resultados básicos de teoria de representações, e aplicamos estes resultados para obter os resultados principais do capítulo.

Também vale notar que calculamos um exemplo concreto (e já conhecido) utili- 
zando o resultado principal do capítulo. 


\section{Capítulo 5}

\section{Referências}

[1] Goodaire, E.G., Jespers, E., Polcino Milies, C., Alternative Loop Rings, NorthHolland Math. Stud., vol. 184, Elsevier, Amsterdam, 1996.

[2] Jespers, E., Leal, G., Paques, A., Central idempotents in the rational group algebra of a finite nilpotent group, Journal of Algebra and Its Applications Vol. 2, No. 1 (2003) 57-62.

[3] Niven, I., Zuckerman, H.S., Montgomery, H.L., An Introduction to the Theory of Numbers, fifth ed., Wiley, 1991.

[4] Polcino Milies, C, Ferraz, R.A., Idempotents in group algebras and minimal abelian codes, Finite Fields and Their Applications 13 (2007) 382-393.

[5] Polcino Milies, C, Sehgal, S.K., An Introduction to Group Rings, Kluwer Academic, Dordrecht, 2002.

[6] Pruthi, M., Arora, S.K., Minimal codes of prime power length, Finite Fields Appl. 3 (1997) 99-113.

[7] Robinson, D.J.S., A Course in the Theory of Groups, Grad. Texts in Math., Springer-Verlag, New York, 1993. 
[8] Roth, R., Introduction to Coding Theory, Cambridge University Press, New York, 2006.

[9] Rotman, J.J., An Introduction to the Theory of Groups, fourth ed., Grad. Texts in Math., vol. 148, Springer-Verlag, New York, 1995. 


\section{Índice Remissivo}

Anéis de Grupos, 3, 5

semissimples, 6, 7, 19, 20

Códigos, 19

abelianos, 19, 24

abelianos minimais, 31

cíclicos, 11

cíclicos minimais, 24, 26

ideais minimais

dimensão e distância mínima, 33

polinômio de verificação, 12

polinômio gerador, 11-13, 36

Caracteres em Grupos Abelianos Finitos, Teorema de Wedderburn-Artin, 6
Matrizes Unitriangulares, 16 tamanho 3, 59

Representações de Grupos, 39

fiel, 40, 44

irredutível, 40, 44

Série Central, 16

ascendente, 14

descendente, 14

Teorema de Mascke, 6

Teorema de Perlis-Walker, 8

9

Centros Superiores, 14

Classe q-Ciclotômica, 20

Grupos Nilpotentes, 14

centro, 15

classe de nilpotência, 15

idempotentes primitivos de álgebras, 50

p-grupos, 16

Idempotentes Primitivos, 8, 25, 26, 32, 47

Lema de Schür, 40 